LMU-ASC 19/12

FLAVOUR(267104)-ERC-9

March 2012

\title{
Effective Theory of a Dynamically Broken Electroweak Standard Model at NLO
}

\author{
Gerhard Buchalla and Oscar Catà \\ Ludwig-Maximilians-Universität München, Fakultät für Physik, \\ Arnold Sommerfeld Center for Theoretical Physics, D-80333 München, Germany
}

\begin{abstract}
We consider the Standard Model as an effective theory at the weak scale $v$ of a generic new strong interaction that dynamically breaks electroweak symmetry at the energy scale $\Lambda \sim$ (few) TeV. Assuming only the minimal field content with the Standard Model fermions and gauge bosons, but without a light Higgs particle, we construct the complete Lagrangian through next-to-leading order, that is, including terms of order $v^{2} / \Lambda^{2}$. The systematics behind this expansion is clarified. Although similar to chiral perturbation theory, it is not governed by the dimension of operators alone, but depends in an essential way on the loop expansion. Power-counting formulas are derived that indicate the classes of operators required at the next-to-leading order. The complete set of operators at leading and next-to-leading order is then listed, based on the restrictions implied by the Standard-Model gauge symmetries. We recover the well-known operators discussed in the literature in connection with the electroweak chiral Lagrangian and in similar contexts, but we collect a complete and systematic list of all terms through order $v^{2} / \Lambda^{2}$. This includes some operators not discussed in explicit terms before. We also show that a few of the previously considered operators can be eliminated via the equations of motion. As another important result we confirm the known list of dimension-6 operators in the Standard Model with an elementary Higgs doublet, essentially as a special case of our scenario.
\end{abstract}

PACS: 11.10.Gh, 11.15.Ex, 12.39.Fe 


\section{Introduction}

The detection of the $W$ and $Z$ gauge bosons almost thirty years ago established that the electroweak interactions are successfully described by a $S U(2)_{L} \otimes U(1)_{Y}$ gauge group spontaneously broken to $U(1)_{Q}$. However, while the Higgs mechanism is certainly at work, the precise way in which the electroweak symmetry is broken remains a mystery. Even whether the underlying dynamics is weakly or strongly coupled remains unclear.

The simplest option for the weakly-coupled scenario is the introduction of a fundamental scalar doublet. Besides the required 3 Goldstone bosons, one obtains a massive scalar field, the (Standard Model) Higgs boson, whose mass has to be taken as a free parameter. Fundamental scalar particles however lead to problems of naturalness, and in order to make the theory meaningful an additional stabilization mechanism has to be invoked. Supersymmetry still stands as the most solid theoretical framework to explain the lightness of a weakly-coupled Higgs.

The alternative is a scenario with dynamical symmetry breaking. In this case spontaneous symmetry breaking is triggered by a condensate, which is generated by new interactions that become strongly coupled at the electroweak scale. This scenario is akin to how chiral symmetry is broken in QCD. Therefore, one of the distinctive features of strongly-coupled scenarios is compositeness and the existence of a large number of bound states, naturally starting at the TeV scale. In general, strongly-coupled scenarios allow for the presence of a light scalar (with mass around the electroweak scale) if it is interpreted as a pseudo-Goldstone boson of a spontaneously broken symmetry group [1,2] . The appeal of such models is that this (composite) Higgs mass is naturally of the order of the electroweak scale [3].

The search for the origin of electroweak symmetry breaking is currently underway at the LHC. At the time of writing, ATLAS and CMS have already excluded a light Higgs boson for a wide range of masses. The non-excluded area is at present in the range 115-130 GeV, with some intriguing excess around $125 \mathrm{GeV}$, not significant enough to be conclusive $[4,5]$. However, even if the existence of a light Higgs is confirmed, we will still be unable to discern whether weakly-coupled or strongly-coupled scenarios are at work. Additional information at the $\mathrm{TeV}$ scale will be needed. So far, no signals of $\mathrm{TeV}$ particles, be it SUSY partners or bound states of strongly-coupled theories, have been observed.

In this article we will study strongly-coupled scenarios. In the past, starting with Technicolor models, there has been a huge effort in model-building. However, finding a viable UV completion of a strongly-coupled scenario has proven a hard task, especially when it comes to match the low-energy experimental constraints from LEP. In this paper we will follow a model-independent approach. The language we will use is that of Effective Field Theories, with the only assumption that the low energy degrees of freedom reduce to the presently-established particle content of the Standard Model. We will show that such an effective theory is renormalizable order by order in a $1 / \Lambda^{2}$ expansion $(\Lambda \sim 4 \pi v \sim 3 \mathrm{TeV})$, and will list the full set of operators up to next-to-leading order (NLO). This might be considered the minimal version of an effective theory of strongly- 
coupled electroweak symmetry breaking, in the sense that we are including only three Goldstone fields, i.e. the longitudinal modes of the $W$ and $Z$ gauge bosons.

This approach to dynamical electroweak symmetry breaking was first studied in [6], inspired by the methods developed for chiral symmetry breaking in the strong interactions [7]. Over the years there have been different steps towards extending the work of [6], but a systematic classification of operators is still lacking. A first analysis of the NLO counterterms for the gauge boson and Goldstone sectors was given in [8], which was followed by a more systematic treatment of the operator basis in the CP-conserving [9] and CP-violating [10] sectors. The interactions of fermionic operators with Goldstone modes were first considered in $[11,12]$, where the naive dimension-4 operators were listed. This list was soon enlarged to include also scalar and tensor currents $[13,14,15]$. In the absence of experimental constraints, the coefficients of the different operators were initially estimated with specific UV completions, most prominently Technicolor-inspired models. With the advent of LEP 1 and 2, several phenomenological analyses were devoted to constraining the electroweak chiral Lagrangian with electroweak precision tests, mostly through oblique parameters and triple gauge boson couplings (see, for instance, [16] and references therein).

The purpose of this work is twofold: first, we will clarify issues of power counting and provide a consistent framework for the electroweak effective theory. Then we will present a comprehensive classification of operators to NLO in the $1 / \Lambda^{2}$ expansion. While in the gauge boson sector the list of operators was settled long ago [10], in the quark sector a systematic treatment is still needed. For instance, Ref. [14] considered fermion bilinears but dismissed operators proportional to the fermion masses, which is clearly not a suitable approximation for top quark physics. Moreover, a full classification of 4-fermion operators has not been considered so far in the literature. Finally, due care is paid to eliminate possible redundancies and to reduce the number of operators to a minimal basis. In particular, we show that some relations between operators with fermions and gauge bosons can be obtained by direct application of the equations of motion and integration by parts. Those relations have already been pointed out in $[17,18]$ and can be of importance in the study of triple gauge boson couplings [18].

As already mentioned, the framework we introduce in this article assumes a minimal particle content for the Standard Model. It would be interesting to consider extensions that include a (composite) light (pseudo)scalar, if LHC eventually unveils its existence. These extensions would share some similarities with the setting presented in [2].

The present article is organized as follows: in Section 2 we review the leading order Lagrangian for the electroweak interactions in the absence of a light fundamental Higgs boson. The power counting for the effective theory is discussed in Section 3 and then used in Section 4 to provide the full list of operators at next-to-leading order. As a simple illustration of how such operators can be generated from a UV-complete theory, in Section 5 we consider as a toy model the Standard Model with a heavy Higgs. In Section 6 we contrast our basis of operators with the corresponding NLO operators in the presence of a light Higgs boson. Related issues of decoupling and renormalizability are discussed in Section 7. Conclusions are given in Section 8. The power-counting formulas 
are derived in Appendix A. Technical details on the construction of the operator basis at NLO are provided in Appendix B, while Appendix C lists the full set of operators in the unitary gauge.

\section{SM effective Lagrangian at leading order}

The field content of the Standard Model (SM) is specified by the left-handed doublets $q$, $l$ and right-handed singlets $u, d, e$ of quarks and leptons, together with the gauge fields $G, W, B$ of $S U(3)_{C}, S U(2)_{L}$ and $U(1)_{Y}$. The fermion fields are

$$
q(3,2,1 / 6), \quad l(1,2,-1 / 2), \quad u(3,1,2 / 3), \quad d(3,1,-1 / 3), \quad e(1,1,-1)
$$

with their transformation properties under $S U(3)$ and $S U(2)$ and their hypercharge $Y$ indicated in brackets. Each of the fields carries a generation index $p=1,2,3$, which has been suppressed in (1).

The effective theory of the gauge and fermion fields is constructed by writing down all possible Lorentz-invariant operators, composed of these fields, that are singlets under the SM gauge group. Restriction to operators of dimension less or equal to 4 uniquely determines the renormalizable, unbroken part of the SM Lagrangian

$$
\begin{aligned}
\mathcal{L}_{4}= & -\frac{1}{2}\left\langle G_{\mu \nu} G^{\mu \nu}\right\rangle-\frac{1}{2}\left\langle W_{\mu \nu} W^{\mu \nu}\right\rangle-\frac{1}{4} B_{\mu \nu} B^{\mu \nu} \\
& +\bar{q} i \not D q+\bar{l} i \not D l+\bar{u} i \not D u+\bar{d} i \not D d+\bar{e} i \not D e
\end{aligned}
$$

Here and in the following the trace of a matrix $M$ is denoted by $\langle M\rangle$. The covariant derivative of a fermion field $\psi_{L, R}$ is defined as

$$
D_{\mu} \psi_{L}=\partial_{\mu} \psi_{L}+i g W_{\mu} \psi_{L}+i g^{\prime} Y_{\psi_{L}} B_{\mu} \psi_{L}, \quad D_{\mu} \psi_{R}=\partial_{\mu} \psi_{R}+i g^{\prime} Y_{\psi_{R}} B_{\mu} \psi_{R}
$$

The fields are normalized in the canonical way and the fermionic terms can always be chosen to be diagonal in the generation index. We have not written the topological terms $\langle G \tilde{G}\rangle$ and $\langle W \tilde{W}\rangle$. The effective Lagrangian (2) describes physics at the electroweak scale $v=246 \mathrm{GeV}$, assumed to be small in comparison with a new physics scale $\Lambda$.

Next, $S U(2)_{L} \otimes U(1)_{Y}$ is spontaneously broken to the electromagnetic $U(1)_{Q}$. The associated Goldstone bosons can be described by the nonlinear $S U(2)$ matrix field

$$
U=\exp (2 i \Phi / v), \quad \Phi=\varphi^{a} T^{a}=\frac{1}{\sqrt{2}}\left(\begin{array}{cc}
\frac{\varphi^{0}}{\sqrt{2}} & \varphi^{+} \\
\varphi^{-} & -\frac{\varphi^{0}}{\sqrt{2}}
\end{array}\right)
$$

with $T^{a}=T_{a}$ the generators of $S U(2)$. We further suppose that no additional light fields exist, in particular no physical Higgs boson. Assuming that the Goldstone bosons arise from the spontaneous breaking of a global $S U(2)_{L} \otimes S U(2)_{R}$ symmetry to the diagonal subgroup $S U(2)_{V}$, the field $U$ transforms as

$$
U \rightarrow g_{L} U g_{R}^{\dagger}, \quad g_{L, R} \in S U(2)_{L, R}
$$


The transformations $g_{L}$ and the $U(1)_{Y}$ subgroup of $g_{R}$ are gauged, so that the covariant derivative of $U$ is given by

$$
D_{\mu} U=\partial_{\mu} U+i g W_{\mu} U-i g^{\prime} B_{\mu} U T_{3}
$$

The dynamics of the Goldstone bosons can be described in a model-independent way by constructing the most general Lagrangian, built from $U$ and the remaining SM fields, that is consistent with the SM gauge symmetry. In practice this construction is carried out including all operators that contribute up to a given order in $v / \Lambda$, where $\Lambda$ is the effective theory cutoff. This procedure is well-known from chiral perturbation theory [19]. The systematics of this expansion has some peculiar features in the case of the electroweak Standard Model and will be discussed in detail in the next section. Presently we summarize the terms to be included at lowest order in the Lagrangian, that is, terms of the same order in $v / \Lambda$ as the renormalizable part in (2).

To lowest order the Lagrangian describing the Goldstone bosons and their interactions with the SM fields is given by

$$
\mathcal{L}_{U}=\frac{v^{2}}{4}\left\langle D_{\mu} U^{\dagger} D^{\mu} U\right\rangle-v\left(\bar{q} Y_{u} U P_{+} r+\bar{q} Y_{d} U P_{-} r+\bar{l} Y_{e} U P_{-} \eta+\text { h.c. }\right)
$$

where the right-handed quark and lepton fields are collected in $r=(u, d)^{T}$ and $\eta=$ $(\nu, e)^{T}$, respectively. As before, we do not write generation indices explicitly. The Yukawa matrices $Y_{u, d, e}$ are understood to denote the usual matrices in generation space. We define

$$
P_{ \pm} \equiv \frac{1}{2} \pm T_{3}, \quad P_{12} \equiv T_{1}+i T_{2}, \quad P_{21} \equiv T_{1}-i T_{2}
$$

where $P_{12}$ and $P_{21}$ will be needed below.

The first expression in (7) is the leading term, of $\mathcal{O}\left(p^{2}\right)$, in chiral perturbation theory. It contains the kinetic term of the Goldstone fields and the mass term of the weak gauge bosons. It is therefore of the same size $\sim v^{4}$ as the terms in (2) and has to be counted as a leading order contribution. In principle, all terms of $\mathcal{O}\left(p^{2}\right)$ that are invariant under the SM gauge group $S U(2)_{L} \otimes U(1)_{Y}$ have to be included in the effective theory at lowest order. There are only two such terms, the first operator in (7) and

$$
\mathcal{L}_{\beta_{1}}=\beta_{1} v^{2}\left\langle U^{\dagger} D_{\mu} U T_{3}\right\rangle^{2}
$$

In contrast to the first term in (7), the operator in (9) does not respect the global custodial $S U(2)_{L} \otimes S U(2)_{R}$ symmetry $[8,10]$. It contributes to the electroweak $T$-parameter [20], which is strongly restricted by experimental data. This implies that the coefficient $\beta_{1}$ is constrained to be very small $\left(\beta_{1} \ll 1\right)$ and suggests that the custodial symmetry is largely respected by the underlying dynamics of electroweak symmetry breaking. If this is the case, the operator (9) can in general still be induced through loop corrections. Then $\beta_{1} v^{2} \sim v^{2} /\left(16 \pi^{2}\right) \sim v^{4} / \Lambda^{2}$ and the contribution in (9) amounts to a next-to-leading order correction. The related power-counting will be discussed in detail in section 3 . We have listed this term here for completeness. 
The last term in (7) gives masses to the SM fermions. Motivated by the case of the top quark, we will count the Yukawa couplings $Y_{f}$ as order one. The fermion mass term then contributes at the same order as the previous terms. The operators in this term are the most general expressions built from fermion bilinears and $U$ fields without derivatives. The Lagrangian $\mathcal{L}_{4}+\mathcal{L}_{U}$ thus defines the leading order of the effective theory for the minimal Standard Model without a light Higgs boson.

At the quantum level, the previous list of leading order operators has to be supplemented with the usual gauge-fixing and Faddeev-Popov terms. Expressions for both of these terms in the covariant $R_{\xi}$ gauges are given in [21]. In this paper, following [6], we will work in the Landau gauge. The main advantage of the Landau gauge for the electroweak effective theory we are considering is that ghost and Goldstone fields decouple. As a result, there is no need to include ghosts in the effective operator basis: only Goldstones coupled to gauge and fermion fields are needed to renormalize the divergences of the leading-order Lagrangian of Eq. (7). Additionally, because of this decoupling, the ghost term in Landau gauge is exactly the one of the Standard Model. Therefore, the presence of the conventional Faddeev-Popov term should be implicitly understood in Eq. (7).

\section{Power counting}

In this section we discuss the systematics of the expansion that defines the effective theory. This expansion cannot be simply organized in terms of the dimension of operators alone. This is clear from the Lagrangian $\mathcal{L}_{4}+\mathcal{L}_{U}$ in (2) and (7), which contains operators of dimension 2, 3 and 4, all contributing at leading order. Also, while (2) is renormalizable, (7) contains interactions that are non-renormalizable (in the traditional sense). Such a theory can still be renormalized (in the modern sense), order by order in a loop expansion, at the expense of introducing at each order a finite number of new counterterms, whose structure is dictated by symmetry. A similar discussion has been given previously in the context of the chiral quark model in [22].

It will be our goal to construct the complete next-to-leading order Lagrangian supplementing the leading order terms in $\mathcal{L}_{4}+\mathcal{L}_{U}$. The next-to-leading order is understood here to include all operators, allowed by the relevant symmetries, with coefficients of order $v^{2} / \Lambda^{2}$ (or $\left.p^{2} / \Lambda^{2}\right)$. A minimal set of operators is defined by the terms required to absorb the ultraviolet divergences generated at one loop by the lowest order Lagrangian $\mathcal{L}_{4}+\mathcal{L}_{U}$

As an example, we may consider the terms in the Lagrangian (7), which induce at one loop the diagram for $\psi_{R} \psi_{R} \rightarrow \psi_{L} \psi_{L}$ scattering shown in Fig. 1. Each vertex $\bar{\psi}_{L} \psi_{R} \varphi^{2}$ has a factor of $y / v$, with $y$ a generic Yukawa coupling. The loop integration produces a factor of $1 /\left(16 \pi^{2}\right)=v^{2} / \Lambda^{2}$, and the four powers of loop momentum $p$ in the numerator are compensated by the four powers of $p$ from the two boson propagators. This leads to 


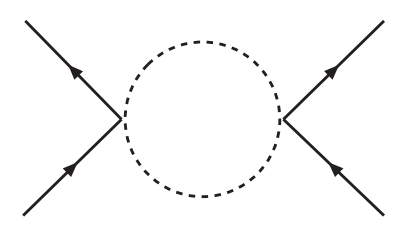

Figure 1: Goldstone-boson loop contributing to fermion-fermion scattering induced by the Yukawa interaction in eq. (7).

the following schematic expression for the diagram

$$
D_{1} \sim \frac{y^{2}}{\Lambda^{2}} p^{0} \bar{\psi}_{L} \psi_{R} \bar{\psi}_{L} \psi_{R}
$$

This result indicates a 4 -fermion amplitude with a logarithmic divergence by power counting. The amplitude matches onto a local, gauge-invariant, dimension- 6 operator $\bar{\psi}_{L} U \psi_{R} \bar{\psi}_{L} U \psi_{R}$, whose coefficient will absorb the ultraviolet divergence. We see that this new operator appears with a natural suppression of order $1 / \Lambda^{2}$. It is therefore part of the next-to-leading order Lagrangian we want to construct.

The example we have just discussed can be generalized. Let us in a first step restrict our attention to the Goldstone-fermion sector, neglecting the gauge fields. This corresponds to taking the leading order Lagrangian $\mathcal{L}_{4}+\mathcal{L}_{U}$ in the gaugeless limit $\left(g_{s}, g\right.$, $\left.g^{\prime} \rightarrow 0\right)$. In this case the interaction vertices are either couplings of an even number $2 i$ of Goldstone bosons with two powers of momentum, contributing a factor of $p^{2} / v^{2 i-2}$, or couplings of $\bar{\psi}_{L} \psi_{R}$ (and h.c.) to an arbitrary number $k$ of bosons, contributing a factor of $y / v^{k-1}$. Considering a general diagram $\mathcal{D}$ with these leading-order vertices, but with any number of loops $L$ and an arbitrary number of external lines, we find the power counting formula

$$
\mathcal{D} \sim \frac{(y v)^{\nu}}{v^{F_{L}+F_{R}-2}} \frac{p^{2 L+2-\nu-\left(F_{L}+F_{R}\right) / 2}}{\Lambda^{2 L}} \bar{\psi}_{L}^{F_{L}^{1}} \psi_{L}^{F_{L}^{2}} \bar{\psi}_{R}^{F_{R}^{1}} \psi_{R}^{F_{R}^{2}}\left(\frac{\varphi}{v}\right)^{B}
$$

where $F_{L, R}=F_{L, R}^{1}+F_{L, R}^{2}$ is the number of external fermions $\psi_{L, R}, B$ is the number of external Goldstone bosons $\varphi$ and $\nu=\sum_{k} \nu_{k}$ is the total number of Yukawa vertices, with $\nu_{k}$ the number of such vertices that have $k$ boson lines. Eq. (11) can be obtained in the standard way by writing down the powers of $y, p, v$ and $\Lambda$ for a general diagram and by eliminating the numbers of internal lines using the familiar topological identities (see Appendix A). Taking $F_{L, R}=\nu=0$, (11) reduces to the corresponding expression for pure chiral perturbation theory

$$
\mathcal{D} \sim \frac{v^{2}}{\Lambda^{2 L}} p^{2 L+2}\left(\frac{\varphi}{v}\right)^{B}
$$

This formula encodes the well-known results on the systematics of chiral perturbation theory: 1) $L$-loop diagrams with only leading-order vertices contribute at $L$ th order in 
the expansion parameter $p^{2} / \Lambda^{2}$ (with $p \sim v$ ). 2) Since the power of $p$ is $2 L+2>0$, an $L$-loop contribution of this type is divergent and requires an order $p^{2 L+2}$ counterterm. According to this pattern only order- $p^{2}$ terms are required at the leading order $(L=0)$ in the chiral Lagrangian. At next-to-leading order $(L=1)$ we need in addition all terms of order $p^{4}$. These are the $S U(2)_{L} \otimes U(1)_{Y}$ gauge invariant operators built from $U$ fields and containing four covariant derivatives, symbolically of the form $U D^{4}$.

When fermions are taken into account in addition to the Goldstone bosons, (11) is the relevant power-counting formula. To find the required counterterms we proceed as before. The order in the expansion in $1 / \Lambda^{2}$ is again determined by $L$. A non-negative power of $p$ signals a divergent diagram. At the same time it gives the number of derivatives in the local operator that is needed to absorb the divergence. (We are assuming the use of dimensional regularization throughout the present paper.) For the next-to-leading order we are mainly interested in, we have $L=1$ and the condition for a counterterm reads

$$
4-\nu-\frac{F_{L}+F_{R}}{2} \geq 0
$$

Since the number of external fermions $F \equiv F_{L}+F_{R}$, and also the number of fermion vertices $\nu$, enters with a negative sign, the number of fermion fields that can appear in the counterterms is limited. Eq. (13) implies that only $F=2$ and $F=4$ are allowed. For $F=6$ (and higher) we need at least $\nu=3$ Yukawa vertices and (13) can no longer be fulfilled. In these cases, the negative power of $p$ indicates an ultraviolet convergent diagram, which is part of the finite radiative corrections predicted at one loop by the lowest order Lagrangian.

The cases that lead to counterterms are then

$$
(F, \nu, 4-\nu-F / 2)=(2,1,2), \quad(2,2,1), \quad(2,3,0), \quad(4,2,0)
$$

The case $(2,3,0)$ corresponds to fermion bilinears with $U$ fields but without derivatives. These are precisely the Yukawa terms already present in the leading-order Lagrangian (7). The new terms are fermion bilinears with one or two derivatives, $(2,2,1),(2,1,2)$, and 4-fermion operators with no derivatives, (4,2,0), all of them with an arbitrary number of $U$ fields. We will denote these three classes schematically as $\psi^{2} U D, \psi^{2} U D^{2}$ and $\psi^{4} U$.

These considerations may be generalized to include interactions with gauge fields. In what follows we will omit ghost fields, which would insure manifest gauge independence, but would not affect the power counting. A generic diagram $\mathcal{D}$ then contains, besides the $n_{i} \varphi^{2 i}$-vertices and $\nu_{k}$ Yukawa interactions $\bar{\psi}_{L(R)} \psi_{R(L)} \varphi^{k}$, a number $m_{l}$ of gaugeboson-Goldstone vertices $X_{\mu} \varphi^{l}, r_{s}$ such vertices of the type $X_{\mu}^{2} \varphi^{s}, x$ quartic gaugeboson vertices $X_{\mu}^{4}, u$ triple-gauge-boson vertices $X_{\mu}^{3}$, and $z_{L}\left(z_{R}\right)$ fermion-gauge-boson interactions $\bar{\psi}_{L(R)} \psi_{L(R)} X_{\mu}$. Here $\psi_{L}\left(\psi_{R}\right), \varphi$ and $X_{\mu}$ denote left-handed (right-handed) fermions, Goldstone bosons and gauge fields, respectively. As discussed in Appendix A, the power-counting for a diagram $\mathcal{D}$ can be summarized by the formula

$$
\mathcal{D} \sim \frac{(y v)^{\nu}(g v)^{m+2 r+2 x+u+z}}{v^{F_{L}+F_{R}-2}} \frac{p^{d}}{\Lambda^{2 L}} \bar{\psi}_{L}^{F_{L}^{1}} \psi_{L}^{F_{L}^{2}} \bar{\psi}_{R}^{F_{R}^{1}} \psi_{R}^{F_{R}^{2}}\left(\frac{X_{\mu \nu}}{v}\right)^{V}\left(\frac{\varphi}{v}\right)^{B}
$$


where the power of $p$ is

$$
d \equiv 2 L+2-\frac{F_{L}+F_{R}}{2}-V-\nu-m-2 r-2 x-u-z
$$

Here $V$ is the number of external gauge-boson lines, $g$ a generic gauge coupling, and we have used $\nu \equiv \sum_{k} \nu_{k}, m \equiv \sum_{l} m_{l}, r \equiv \sum_{s} r_{s}, z \equiv z_{L}+z_{R}$. Eq. (15) generalizes (11), to which it reduces when neglecting the gauge fields, that is, taking $V=m=r=x=u=$ $z=0$. The expression (16) for $d$ is useful, because $F_{L}, F_{R}$ and $V$, as well as the numbers of vertices, all enter with a negative sign. This implies a limit to the number of divergent diagrams at a given order in $L$, similar to the special case of (11). We also note that, as in (11), the number $B$ of external Goldstone bosons enters the power counting formula (15) only through the factor $(\varphi / v)^{B}$. It is irrelevant in particular for the exponent $d$, which counts the powers of momentum. This indicates explicitly that, at any given order in the effective theory, the counterterms contain an arbitrary number of Goldstone fields $U=U(\varphi / v)$.

We will next use (15) and (16) to find the remaining classes of NLO counterterms, which contain at least one gauge-field operator. The cases to be considered are then $L=1, F \geq 0, V \geq 1$.

$F=0, V=1:$ In this case we have

$$
d=3-\nu-m-2 r-2 x-u-z
$$

Since $V=1$, the diagram must contain at least one gauge-field vertex, that is, at least one of $(m, r, x, u, z)$ must be non-zero. This implies $d \leq 2$. The divergent amplitudes then have $d=2,1$ or 0 , and correspond to counterterms of the form $X U D^{2}, X U D$ and $X U$, respectively. No Lorentz scalars can be built from the latter two, which leaves us with the operator class $X U D^{2}$.

$F=0, V=2$ : The exponent of $p$ now reads $d=2-\nu-m-2 r-2 x-u-z$. With two external gauge fields the diagram has at least two single-gauge-field vertices $(m+u+z \geq 2)$, or one double-gauge-field vertex $(r+x \geq 1)$. It follows that $d \leq 0$. For $d=0$ the diagram requires a counterterm, with $F=0, V=2$ and $d=0$ derivatives. This determines its form to be $X^{2} U$.

$F=0, V=3$ : With $d=1-\nu-m-2 r-2 x-u-z$ and at least two vertices required to form a loop diagram, we have $d<0$. The diagrams of this type are convergent. Operators of class $X^{3}$ are therefore not required as counterterms of the nonrenormalizable effective Lagrangian. Representing operators of dimension 6 they could a priori still give (finite) contributions of order $1 / \Lambda^{2}$, that is at NLO. We will return to this and similar cases below. The case of $F=0, V>3$ leads to $d<0$ and to operators beyond the NLO approximation.

$F=2, V=1$ : Here the power-counting formula gives $d=2-\nu-m-2 r-2 x-u-z$. The minimum number of vertices one might consider is two. This can be realized 
when a $\psi^{2} \varphi^{2}$-vertex is connected to an $X_{\mu} \varphi^{2}$-vertex, contracting the $\varphi$ fields to form a one-loop diagram. By inspection, such a diagram is found to be zero. A nonvanishing diagram thus requires at least three vertices, implying $d<0$. The diagram converges and the corresponding operators of class $\psi^{2} U X$ are not needed as counterterms. It is clear from these considerations that even larger numbers for $F$ and $V$ can only lead to $d<0$ and to terms beyond the NLO level.

This completes the enumeration of NLO counterterms. To summarize, power counting allows us to identify the classes of operators we need to include in the next-to-leading order Lagrangian. Denoting by $U$ the presence of any number of Goldstone fields $U$ (or $U^{\dagger}$ ), and by $D^{n}, \psi^{F}, X^{k}$ the numbers $n, F, k$, respectively, of derivatives, fermion fields and gauge-boson field-strength tensors, these classes are schematically given by

$$
U D^{4}, \quad X U D^{2}, \quad X^{2} U, \quad \psi^{2} U D, \quad \psi^{2} U D^{2}, \quad \psi^{4} U
$$

We remark that all the possible classes of dimension-4 operators built from $U, D, X$ and $\psi$ appear as NLO counterterms $\left(U D^{4}, X U D^{2}, X^{2} U, \psi^{2} U D\right)$, but there are also operators of dimension 5 and 6 at the same level $\left(\psi^{2} U D^{2}, \psi^{4} U\right)$.

All operators that are not required as counterterms at NLO are naturally of higher order in $1 / \Lambda^{2}$. This is clear for operators of canonical dimension larger than 6 . These have a coefficient $\sim 1 / \Lambda^{n}, n \geq 3$ on purely dimensional grounds, which is possibly further suppressed by loop factors $1 /\left(16 \pi^{2}\right)=v^{2} / \Lambda^{2}$. A special consideration is needed for operators of dimension 5 and 6 , since those may appear either at NLO $\left(\sim 1 / \Lambda^{2}\right)$, or only at NNLO $\left(\sim 1 / \Lambda^{4}\right)$. The former case is illustrated by operators of the type $\left(\bar{\psi}_{L} U \psi_{R}\right)^{2}$. Examples for the latter case are operators with Goldstone fields and six derivatives (class $\left.U D^{6}\right)$. More systematically, the various classes of such operators of dimension 5 and 6 , which would only appear as counterterms at NNLO, are given by: $U D^{6}, X U D^{4}, X^{2} U D^{2}$, $X^{3} U, \psi^{2} U D^{3}, \psi^{2} U X D$, and $\psi^{2} U X$. We will not provide a complete classification of those operators, but we would like to comment on a few relevant aspects.

To some extent the actual size of the coefficient of a given operator depends on the UV completion of the theory. It is, however, natural to expect that a NNLO counterterm will have a coefficient no larger than $\mathcal{O}\left(1 / \Lambda^{4}\right)$. As an illustration we may consider the operator $G_{\mu \nu} G^{\mu \nu}\left\langle D_{\lambda} U^{\dagger} D^{\lambda} U\right\rangle$, which belongs to the class $X^{2} U D^{2}$ of NNLO counterterms. In a model with a heavy Higgs boson, this operator would be generated by Higgs exchange between the Higgs-Goldstone couplings and the Higgs-gluon-gluon vertex. The latter would contain a loop factor $v^{2} / \Lambda^{2}$, while the Higgs propagator would contribute a factor of $1 / m_{h}^{2} \sim 1 / \Lambda^{2}$, leading to an $\mathcal{O}\left(1 / \Lambda^{4}\right)$ coefficient. A similar consideration applies to the operators $\psi^{2} U X$ and $X^{3} U$, which are typically loop induced [23]. The NNLO classes $\psi^{2} U X$ and $X^{3} U$ are special in that they directly correspond to dimension-6 operators appearing at NLO in the conventional Standard Model with a linear Higgs doublet. Since we want to compare this scenario with our framework later on, we will also list below the few operators of classes $\psi^{2} U X$ and $X^{3}$ (but not $X^{3} U$ ).

As we have seen above, 4 -fermion operators of the form $\bar{\psi}_{L} U \psi_{R} \bar{\psi}_{L} U \psi_{R}$ arise as counterterms at next-to-leading order in the effective Lagrangian and thus have to be 
included in our operator basis. We are thus led to consider the entire class of 4-fermion operators, which may include an arbitrary number of $U$ fields. However, not all of these operators are required as counterterms of the Lagrangian $\mathcal{L}_{4}+\mathcal{L}_{U}$ at next-to-leading order. An example is the case of $\bar{\psi}_{L} \gamma^{\mu} \psi_{L} \bar{\psi}_{L} \gamma_{\mu} \psi_{L}$, whose one-loop coefficient is finite by power counting $\left(F_{R}=0, F_{L}=\nu=4\right.$ in (13)). It is then natural to expect this term only at NNLO $\sim 1 / \Lambda^{4}$. However, depending on the details of the physics at the $\mathrm{TeV}$ scale, such an operator could possibly be generated through tree-level exchange of a heavy boson. In this case, the coefficient could be of order $1 / \Lambda^{2}$ and the operator would enter already at NLO, giving a finite contribution at this level. We will not distinguish explicitly between NLO and NNLO counterterms in the sector $\psi^{4} U$ and include the complete list of 4 -fermion operators in our basis, except those that violate baryon $(B)$ or lepton number $(L)$. We will briefly return to such operators in section 4.7.

We finally remark that, independent of the nonrenormalizable interactions organized into a $1 / \Lambda^{2}$ expansion, the usual corrections from the renormalizable part of the Standard Model can be computed, in principle to arbitrary order. This applies e.g. to corrections from perturbative QCD.

In the following section we will provide the complete list of NLO operators for each of the classes in (18). As discussed above, we will also consider the operators $X^{3}$ and $\psi^{2} U X$.

\section{SM effective Lagrangian at next-to-leading order}

The next-to-leading-order effective Lagrangian of the Standard Model with dynamically broken electroweak symmetry and minimal particle content can be written as

$$
\mathcal{L}=\mathcal{L}_{4}+\mathcal{L}_{U}+\mathcal{L}_{\beta_{1}}+\sum_{i} c_{i} \frac{v^{6-d_{i}}}{\Lambda^{2}} \mathcal{O}_{i}
$$

Here $\mathcal{L}_{4}+\mathcal{L}_{U}$ is the leading order Lagrangian and $\mathcal{L}_{\beta_{1}}$ the custodial-symmetry breaking, dimension-2 operator of section 2. As discussed there, $\mathcal{L}_{\beta_{1}}$ can be treated as an NLO correction. Apart from this term, the full set of NLO operators is denoted by the $\mathcal{O}_{i}$ in (19). They come with a suppression by two powers of the cutoff $\Lambda \approx 4 \pi v$ and have dimensionless coefficients $c_{i}$, which are naturally of order unity. $d_{i}$ is the canonical dimension of the operator $\mathcal{O}_{i}$. We will in general assume conservation of baryon and lepton number ( $B$ and $L$ ) in (19) and return to a brief consideration of $B$ and $L$ violating effects in section 4.7 .

In the following we list all NLO operators $\mathcal{O}_{i}$, grouped according to the subsets introduced at the end of section 3. Most of the operators are hermitean. For the others, independent hermitean conjugate versions are always understood and have not been listed separately. We will comment on some important points here but will otherwise relegate details on the derivation to Appendix B. 


\section{$4.1 U D^{4}$ terms}

The operators of this class correspond to the $\mathcal{O}\left(p^{4}\right)$ chiral-Lagrangian terms already listed by Longhitano [9]. They read

$$
\begin{aligned}
\mathcal{O}_{D 1} & =\left\langle D_{\mu} U^{\dagger} D^{\mu} U\right\rangle^{2} \\
\mathcal{O}_{D 2} & =\left\langle D_{\mu} U^{\dagger} D_{\nu} U\right\rangle\left\langle D^{\mu} U^{\dagger} D^{\nu} U\right\rangle \\
\mathcal{O}_{D 3} & =\left(\left\langle U^{\dagger} D_{\mu} U T_{3}\right\rangle\left\langle U^{\dagger} D^{\mu} U T_{3}\right\rangle\right)^{2} \\
\mathcal{O}_{D 4} & =\left\langle U^{\dagger} D_{\mu} U T_{3}\right\rangle\left\langle U^{\dagger} D^{\mu} U T_{3}\right\rangle\left\langle D_{\nu} U^{\dagger} D^{\nu} U\right\rangle \\
\mathcal{O}_{D 5} & =\left\langle U^{\dagger} D_{\mu} U T_{3}\right\rangle\left\langle U^{\dagger} D_{\nu} U T_{3}\right\rangle\left\langle D^{\mu} U^{\dagger} D^{\nu} U\right\rangle
\end{aligned}
$$

\section{2 $X U D^{2}$ and $X^{2} U$ terms}

The CP-even operators are $[9,10]$

$$
\begin{aligned}
& \mathcal{O}_{X U 1}=g^{\prime} g B_{\mu \nu}\left\langle U^{\dagger} W^{\mu \nu} U T_{3}\right\rangle \\
& \mathcal{O}_{X U 2}=g^{2}\left\langle U^{\dagger} W_{\mu \nu} U T_{3}\right\rangle\left\langle U^{\dagger} W^{\mu \nu} U T_{3}\right\rangle \\
& \mathcal{O}_{X U 3}=g \varepsilon^{\mu \nu \lambda \rho}\left\langle U^{\dagger} W_{\mu \nu} D_{\lambda} U\right\rangle\left\langle U^{\dagger} D_{\rho} U T_{3}\right\rangle
\end{aligned}
$$

The list of $X^{2} U$ and $X U D^{2}$ operators also includes three independent CP-odd terms $[10]$

$$
\begin{aligned}
& \mathcal{O}_{X U 4}=g^{\prime} g \varepsilon^{\mu \nu \lambda \rho} B_{\mu \nu}\left\langle U^{\dagger} W_{\lambda \rho} U T_{3}\right\rangle \\
& \mathcal{O}_{X U 5}=g^{2} \varepsilon^{\mu \nu \lambda \rho}\left\langle U^{\dagger} W_{\mu \nu} U T_{3}\right\rangle\left\langle U^{\dagger} W_{\lambda \rho} U T_{3}\right\rangle \\
& \mathcal{O}_{X U 6}=g\left\langle U^{\dagger} W_{\mu \nu} D^{\mu} U\right\rangle\left\langle U^{\dagger} D^{\nu} U T_{3}\right\rangle
\end{aligned}
$$

We note that three more CP-even operators that had been listed in [10] can be expressed in terms of other operators in the complete basis and are thus redundant $[17$, 18]. This follows from applying the equations of motion and discarding total derivatives. For example, the first of these operators is

$$
\begin{aligned}
& B_{\mu \nu}\left\langle D^{\mu} U^{\dagger} D^{\nu} U T_{3}\right\rangle \equiv \\
& \partial^{\mu}\left[B_{\mu \nu}\left\langle U^{\dagger} D^{\nu} U T_{3}\right\rangle\right]-\partial^{\mu} B_{\mu \nu}\left\langle U^{\dagger} D^{\nu} U T_{3}\right\rangle-\frac{1}{2} B_{\mu \nu}\left\langle U^{\dagger}\left(D^{\mu} D^{\nu}-D^{\nu} D^{\mu}\right) U T_{3}\right\rangle= \\
& \frac{i g^{\prime}}{2} v^{2}\left\langle U^{\dagger} D^{\nu} U T_{3}\right\rangle^{2}-g^{\prime} \sum_{\psi} Y_{\psi} \bar{\psi} \gamma_{\nu} \psi\left\langle U^{\dagger} D^{\nu} U T_{3}\right\rangle-\frac{i g}{2} B_{\mu \nu}\left\langle U^{\dagger} W^{\mu \nu} U T_{3}\right\rangle+\frac{i g^{\prime}}{4} B_{\mu \nu} B^{\mu \nu}
\end{aligned}
$$

where the second equality holds up to the total derivative. Similar considerations apply to the operators

$$
\left\langle U^{\dagger} W_{\mu \nu} U T_{3}\right\rangle\left\langle D^{\mu} U^{\dagger} D^{\nu} U T_{3}\right\rangle, \quad\left\langle U^{\dagger} W_{\mu \nu} U D^{\mu} U^{\dagger} D^{\nu} U\right\rangle
$$

as will be further discussed in Appendix B. 


\section{$4.3 \psi^{2} U D$ terms}

For the quark and lepton sectors one finds in this class:

$$
\begin{array}{cc}
\mathcal{O}_{\psi V 1}=\bar{q} \gamma^{\mu} q\left\langle U^{\dagger} i D_{\mu} U T_{3}\right\rangle, & \mathcal{O}_{\psi V 4}=\bar{u} \gamma^{\mu} u\left\langle U^{\dagger} i D_{\mu} U T_{3}\right\rangle \\
\mathcal{O}_{\psi V 2}=\bar{q} \gamma^{\mu} U T_{3} U^{\dagger} q\left\langle U^{\dagger} i D_{\mu} U T_{3}\right\rangle, & \mathcal{O}_{\psi V 5}=\bar{d} \gamma^{\mu} d\left\langle U^{\dagger} i D_{\mu} U T_{3}\right\rangle \\
\mathcal{O}_{\psi V 3}=\bar{q} \gamma^{\mu} U P_{12} U^{\dagger} q\left\langle U^{\dagger} i D_{\mu} U P_{21}\right\rangle \text { (h.c.), } & \mathcal{O}_{\psi V 6}=\bar{u} \gamma^{\mu} d\left\langle U^{\dagger} i D_{\mu} U P_{21}\right\rangle \text { (h.c.) } \\
\mathcal{O}_{\psi V 7}=\bar{l} \gamma^{\mu} l\left\langle U^{\dagger} i D_{\mu} U T_{3}\right\rangle, & \mathcal{O}_{\psi V 10}=\bar{e} \gamma^{\mu} e\left\langle U^{\dagger} i D_{\mu} U T_{3}\right\rangle \\
\mathcal{O}_{\psi V 8}=\bar{l} \gamma^{\mu} U T_{3} U^{\dagger} l\left\langle U^{\dagger} i D_{\mu} U T_{3}\right\rangle & \\
\mathcal{O}_{\psi V 9}=\bar{l} \gamma^{\mu} U P_{12} U^{\dagger} l\left\langle U^{\dagger} i D_{\mu} U P_{21}\right\rangle & \text { (h.c.) }
\end{array}
$$

These operators are equivalent to those given as $\mathcal{L}_{4}^{1}, \ldots, \mathcal{L}_{4}^{6}$ in eq. (20) of [11]. The term $\mathcal{L}_{4}^{7}$ listed there can be eliminated using the equations of motion, as already noted in $[14]$.

In general, $\mathcal{O}_{\psi V 3}$ and its hermitean conjugate are two independent operators. If the coefficients are real, only the combination $\mathcal{O}_{\psi V 3}+\mathcal{O}_{\psi V 3}^{\dagger}$ can appear in the Lagrangian. This combination can be eliminated in favour of the simpler operator

$$
\bar{q} \gamma^{\mu} i D_{\mu} U U^{\dagger} q \equiv \mathcal{O}_{\psi V 3}+\mathcal{O}_{\psi V 3}^{\dagger}+2 \mathcal{O}_{\psi V 2}
$$

Similar considerations apply to $\mathcal{O}_{\psi V 9}$, where

$$
\bar{l} \gamma^{\mu} i D_{\mu} U U^{\dagger} l \equiv \mathcal{O}_{\psi V 9}+\mathcal{O}_{\psi V 9}^{\dagger}+2 \mathcal{O}_{\psi V 8}
$$

\section{$4.4 \psi^{2} U D^{2}$ terms}

The operators with fermionic scalar currents are:

$$
\begin{aligned}
\mathcal{O}_{\psi S 1,2} & =\bar{q} U P_{ \pm} r\left\langle D_{\mu} U^{\dagger} D^{\mu} U\right\rangle \\
\mathcal{O}_{\psi S 3,4} & =\bar{q} U P_{ \pm} r\left\langle U^{\dagger} D_{\mu} U T_{3}\right\rangle^{2} \\
\mathcal{O}_{\psi S 5} & =\bar{q} U P_{12} r\left\langle U^{\dagger} D_{\mu} U P_{21}\right\rangle\left\langle U^{\dagger} D^{\mu} U T_{3}\right\rangle \\
\mathcal{O}_{\psi S 6} & =\bar{q} U P_{21} r\left\langle U^{\dagger} D_{\mu} U P_{12}\right\rangle\left\langle U^{\dagger} D^{\mu} U T_{3}\right\rangle \\
\mathcal{O}_{\psi S 7} & =\bar{l} U P_{-} \eta\left\langle D_{\mu} U^{\dagger} D^{\mu} U\right\rangle \\
\mathcal{O}_{\psi S 8} & =\bar{l} U P_{-} \eta\left\langle U^{\dagger} D_{\mu} U T_{3}\right\rangle^{2} \\
\mathcal{O}_{\psi S 9} & =\bar{l} U P_{12} \eta\left\langle U^{\dagger} D_{\mu} U P_{21}\right\rangle\left\langle U^{\dagger} D^{\mu} U T_{3}\right\rangle
\end{aligned}
$$


The operators with fermionic tensor currents are:

$$
\begin{aligned}
\mathcal{O}_{\psi T 1} & =\bar{q} \sigma^{\mu \nu} U P_{12} r\left\langle U^{\dagger} D_{\mu} U P_{21}\right\rangle\left\langle U^{\dagger} D_{\nu} U T_{3}\right\rangle \\
\mathcal{O}_{\psi T 2} & =\bar{q} \sigma^{\mu \nu} U P_{21} r\left\langle U^{\dagger} D_{\mu} U P_{12}\right\rangle\left\langle U^{\dagger} D_{\nu} U T_{3}\right\rangle \\
\mathcal{O}_{\psi T 3,4} & =\bar{q} \sigma^{\mu \nu} U P_{ \pm} r\left\langle U^{\dagger} D_{\mu} U P_{12}\right\rangle\left\langle U^{\dagger} D_{\nu} U P_{21}\right\rangle \\
\mathcal{O}_{\psi T 5} & =\bar{l} \sigma^{\mu \nu} U P_{12} \eta\left\langle U^{\dagger} D_{\mu} U P_{21}\right\rangle\left\langle U^{\dagger} D_{\nu} U T_{3}\right\rangle \\
\mathcal{O}_{\psi T 6} & =\bar{l} \sigma^{\mu \nu} U P_{-} \eta\left\langle U^{\dagger} D_{\mu} U P_{12}\right\rangle\left\langle U^{\dagger} D_{\nu} U P_{21}\right\rangle
\end{aligned}
$$

\section{5 $\quad \psi^{4} U$ and $\psi^{4}$ terms}

The dimension- 6 operators with four fermion fields and an arbitrary number of Goldstone fields $U$ can be classified as follows. First, there are the 4-fermion operators without $U$ fields (class $\psi^{4}$ ), which have already been listed in [24]. In this case the 4 fermions necessarily have vanishing total hypercharge, $Y\left(\psi^{4}\right)=0$. A new class arises when these operators are dressed with zero-hypercharge combinations of $U$ fields that are contracted in all possible $S U(2)$-invariant ways with the fermion doublets. Beyond this possibility we have to consider operators with 4 fermions that have a combined hypercharge of $Y\left(\psi^{4}\right)= \pm 1 / 2, \pm 1, \pm 3 / 2$ and \pm 2 , because these values can be compensated by the inclusion of $U$ fields. Since $U$ carries $Y= \pm 1 / 2$ and we can have at most 4 factors of $U$ to form $S U(2)$ invariants with the maximum number of $4 S U(2)$-doublet fermions, operators with $\left|Y\left(\psi^{4}\right)\right|>2$ are not allowed. Listing all 4-fermion combinations, it turns out that the classes $Y\left(\psi^{4}\right)= \pm 1 / 2, \pm 3 / 2$ and \pm 2 contain only baryon or lepton number violating operators. This leaves us with $\psi^{4} U$ operators that either have $Y\left(\psi^{4}\right)=0$ or \pm 1 . In the following all fermion fields carry again an independent generation index, which is suppressed in our notation. The generators of $S U(2)_{L}\left(S U(3)_{C}\right)$ are denoted by $T^{a}\left(T^{A}\right)$. Hermitian conjugate versions of the operators listed below are understood.

The $\psi^{4}$ operators [24] can be divided into four groups: $\bar{L} L \bar{L} L, \bar{R} R \bar{R} R, \bar{L} L \bar{R} R$, and $\bar{L} R \bar{L} R$, according to the handedness of the fermions.

The $\bar{L} L \bar{L} L$ operators are

$$
\begin{gathered}
\mathcal{O}_{L L 1}=\bar{q} \gamma^{\mu} q \bar{q} \gamma_{\mu} q, \quad \mathcal{O}_{L L 2}=\bar{q} \gamma^{\mu} T^{a} q \bar{q} \gamma_{\mu} T^{a} q \\
\mathcal{O}_{L L 3}=\bar{q} \gamma^{\mu} q \bar{l} \gamma_{\mu} l, \quad \mathcal{O}_{L L 4}=\bar{q} \gamma^{\mu} T^{a} q \bar{l} \gamma_{\mu} T^{a} l \\
\mathcal{O}_{L L 5}=\bar{l} \gamma^{\mu} l \bar{l} \gamma_{\mu} l
\end{gathered}
$$

The $\bar{R} R \bar{R} R$ operators are

$$
\begin{aligned}
& \mathcal{O}_{R R 1}=\bar{u} \gamma^{\mu} u \bar{u} \gamma_{\mu} u, \quad \mathcal{O}_{R R 2}=\bar{d} \gamma^{\mu} d \bar{d} \gamma_{\mu} d \\
& \mathcal{O}_{R R 3}=\bar{u} \gamma^{\mu} u \bar{d} \gamma_{\mu} d, \quad \mathcal{O}_{R R 4}=\bar{u} \gamma^{\mu} T^{A} u \bar{d} \gamma_{\mu} T^{A} d \\
& \mathcal{O}_{R R 5}=\bar{u} \gamma^{\mu} u \bar{e} \gamma_{\mu} e, \quad \mathcal{O}_{R R 6}=\bar{d} \gamma^{\mu} d \bar{e} \gamma_{\mu} e
\end{aligned}
$$




$$
\mathcal{O}_{R R 7}=\bar{e} \gamma^{\mu} e \bar{e} \gamma_{\mu} e
$$

The $\bar{L} L \bar{R} R$ operators are

$$
\begin{array}{cc}
\mathcal{O}_{L R 1}=\bar{q} \gamma^{\mu} q \bar{u} \gamma_{\mu} u, & \mathcal{O}_{L R 2}=\bar{q} \gamma^{\mu} T^{A} q \bar{u} \gamma_{\mu} T^{A} u \\
\mathcal{O}_{L R 3}=\bar{q} \gamma^{\mu} q \bar{d} \gamma_{\mu} d, \quad \mathcal{O}_{L R 4}=\bar{q} \gamma^{\mu} T^{A} q \bar{d} \gamma_{\mu} T^{A} d \\
\mathcal{O}_{L R 5}=\bar{u} \gamma^{\mu} u \bar{l} \gamma_{\mu} l, & \mathcal{O}_{L R 6}=\bar{d} \gamma^{\mu} d \bar{l} \gamma_{\mu} l \\
\mathcal{O}_{L R 7}=\bar{q} \gamma^{\mu} q \bar{e} \gamma_{\mu} e, & \mathcal{O}_{L R 8}=\bar{l} \gamma^{\mu} l \bar{e} \gamma_{\mu} e \\
\mathcal{O}_{L R 9}=\bar{q} \gamma^{\mu} l \bar{e} \gamma_{\mu} d
\end{array}
$$

The $\bar{L} R \bar{L} R$ operators are

$$
\begin{array}{ll}
\mathcal{O}_{S T 1}=\varepsilon_{i j} \bar{q}^{i} u \bar{q}^{j} d, & \mathcal{O}_{S T 2}=\varepsilon_{i j} \bar{q}^{i} T^{A} u \bar{q}^{j} T^{A} d \\
\mathcal{O}_{S T 3}=\varepsilon_{i j} \bar{q}^{i} u \bar{l}^{j} e, & \mathcal{O}_{S T 4}=\varepsilon_{i j} \bar{q}^{i} \sigma^{\mu \nu} u \bar{l}^{j} \sigma_{\mu \nu} e
\end{array}
$$

The $\bar{L} L \bar{L} L$ operators with $U$ fields are ( $\alpha, \beta$ denote colour indices)

$$
\begin{aligned}
& \mathcal{O}_{L L 6}=\bar{q} \gamma^{\mu} U T_{3} U^{\dagger} q \bar{q} \gamma_{\mu} U T_{3} U^{\dagger} q, \quad \mathcal{O}_{L L 7}=\bar{q} \gamma^{\mu} U T_{3} U^{\dagger} q \bar{q} \gamma_{\mu} q \\
& \mathcal{O}_{L L 8}=\bar{q}_{\alpha} \gamma^{\mu} U T_{3} U^{\dagger} q_{\beta} \bar{q}_{\beta} \gamma_{\mu} U T_{3} U^{\dagger} q_{\alpha}, \quad \mathcal{O}_{L L 9}=\bar{q}_{\alpha} \gamma^{\mu} U T_{3} U^{\dagger} q_{\beta} \bar{q}_{\beta} \gamma_{\mu} q_{\alpha} \\
& \mathcal{O}_{L L 10}=\bar{q} \gamma^{\mu} U T_{3} U^{\dagger} q \bar{l} \gamma_{\mu} U T_{3} U^{\dagger} l \\
& \mathcal{O}_{L L 11}=\bar{q} \gamma^{\mu} U T_{3} U^{\dagger} q \bar{l} \gamma_{\mu} l, \quad \mathcal{O}_{L L 12}=\bar{q} \gamma^{\mu} q \bar{l} \gamma_{\mu} U T_{3} U^{\dagger} l \\
& \mathcal{O}_{L L 13}=\bar{q} \gamma^{\mu} U T_{3} U^{\dagger} l \bar{l} \gamma_{\mu} U T_{3} U^{\dagger} q, \quad \mathcal{O}_{L L 14}=\bar{q} \gamma^{\mu} U T_{3} U^{\dagger} l \bar{l} \gamma_{\mu} q \\
& \mathcal{O}_{L L 15}=\bar{l} \gamma^{\mu} U T_{3} U^{\dagger} l \bar{l} \gamma_{\mu} U T_{3} U^{\dagger} l, \quad \mathcal{O}_{L L 16}=\bar{l} \gamma^{\mu} U T_{3} U^{\dagger} l \bar{l} \gamma_{\mu} l
\end{aligned}
$$

The $\bar{L} L \bar{R} R$ operators with $U$ fields are

$$
\begin{array}{cc}
\mathcal{O}_{L R 10}=\bar{q} \gamma^{\mu} U T_{3} U^{\dagger} q \bar{u} \gamma_{\mu} u, & \mathcal{O}_{L R 11}=\bar{q} \gamma^{\mu} T^{A} U T_{3} U^{\dagger} q \bar{u} \gamma_{\mu} T^{A} u \\
\mathcal{O}_{L R 12}=\bar{q} \gamma^{\mu} U T_{3} U^{\dagger} q \bar{d} \gamma_{\mu} d, & \mathcal{O}_{L R 13}=\bar{q} \gamma^{\mu} T^{A} U T_{3} U^{\dagger} q \bar{d} \gamma_{\mu} T^{A} d \\
\mathcal{O}_{L R 14}=\bar{u} \gamma^{\mu} u \bar{l} \gamma_{\mu} U T_{3} U^{\dagger} l, & \mathcal{O}_{L R 15}=\bar{d} \gamma^{\mu} d \bar{l} \gamma_{\mu} U T_{3} U^{\dagger} l \\
\mathcal{O}_{L R 16}=\bar{q} \gamma^{\mu} U T_{3} U^{\dagger} q \bar{e} \gamma_{\mu} e, & \mathcal{O}_{L R 17}=\bar{l} \gamma^{\mu} U T_{3} U^{\dagger} l \bar{e} \gamma_{\mu} e \\
\mathcal{O}_{L R 18}=\bar{q} \gamma^{\mu} U T_{3} U^{\dagger} l \bar{e} \gamma_{\mu} d
\end{array}
$$

The $\bar{L} R \bar{L} R$ operators with $U$ fields are

$$
\begin{aligned}
\mathcal{O}_{S T 5}=\bar{q} U P_{+} r \bar{q} U P_{-} r, & & \mathcal{O}_{S T 6}=\bar{q} U P_{21} r \bar{q} U P_{12} r \\
\mathcal{O}_{S T 7}=\bar{q} U P_{+} T^{A} r \bar{q} U P_{-} T^{A} r, & & \mathcal{O}_{S T 8}=\bar{q} U P_{21} T^{A} r \bar{q} U P_{12} T^{A} r \\
\mathcal{O}_{S T 9}=\bar{q} U P_{+} r \bar{l} U P_{-} \eta, & & \mathcal{O}_{S T 10}=\bar{q} U P_{21} r \bar{l} U P_{12} \eta \\
\mathcal{O}_{S T 11}=\bar{q} \sigma^{\mu \nu} U P_{+} r \bar{l} \sigma_{\mu \nu} U P_{-} \eta, & & \mathcal{O}_{S T 12}=\bar{q} \sigma^{\mu \nu} U P_{21} r \bar{l} \sigma_{\mu \nu} U P_{12} \eta
\end{aligned}
$$


Finally, the $\psi^{4} U$ operators with $Y\left(\psi^{4}\right)= \pm 1$ are

$$
\begin{gathered}
\mathcal{O}_{F Y 1}=\bar{q} U P_{+} r \bar{q} U P_{+} r, \quad \mathcal{O}_{F Y 2}=\bar{q} U P_{+} T^{A} r \bar{q} U P_{+} T^{A} r \\
\mathcal{O}_{F Y 3}=\bar{q} U P_{-} r \bar{q} U P_{-} r, \quad \mathcal{O}_{F Y 4}=\bar{q} U P_{-} T^{A} r \bar{q} U P_{-} T^{A} r \\
\mathcal{O}_{F Y 5}=\bar{q} U P_{-} r \bar{r} P_{+} U^{\dagger} q, \quad \mathcal{O}_{F Y 6}=\bar{q} U P_{-} T^{A} r \bar{r} P_{+} U^{\dagger} T^{A} q \\
\mathcal{O}_{F Y 7}=\bar{q} U P_{-} r \bar{l} U P_{-} \eta, \quad \mathcal{O}_{F Y 8}=\bar{q} \sigma^{\mu \nu} U P_{-} r \bar{l} \sigma_{\mu \nu} U P_{-} \eta \\
\mathcal{O}_{F Y 9}=\bar{l} U P_{-} \eta \bar{r} P_{+} U^{\dagger} q \\
\mathcal{O}_{F Y 10}=\bar{l} U P_{-} \eta \bar{l} U P_{-} \eta \\
\mathcal{O}_{F Y 11}=\bar{l} U P_{-} r \bar{r} P_{+} U^{\dagger} l
\end{gathered}
$$

\section{6 $\quad X^{3}$ and $\psi^{2} U X$ terms}

The operators $X^{3}$, built from 3 factors of field-strength tensors, are not required as counterterms at next-to-leading order. Being of dimension 6 , they are suppressed by two powers of the heavy mass scale $\Lambda$. A loop suppression will bring the coefficients further down to the NNLO level $\sim 1 / \Lambda^{4}$. Only if they could be induced at tree level these operators would give (finite) contributions at NLO. We will include them for completeness, as discussed in 3 . In class $X^{3}$, there are only four operators [24,25]

$$
\begin{array}{cc}
\mathcal{O}_{X 1}=f^{A B C} G_{\mu}^{A \nu} G_{\nu}^{B \rho} G_{\rho}^{C \mu}, & \mathcal{O}_{X 2}=f^{A B C} \tilde{G}_{\mu}^{A \nu} G_{\nu}^{B \rho} G_{\rho}^{C \mu} \\
\mathcal{O}_{X 3}=\varepsilon^{a b c} W_{\mu}^{a \nu} W_{\nu}^{b \rho} W_{\rho}^{c \mu}, & \mathcal{O}_{X 4}=\varepsilon^{a b c} \tilde{W}_{\mu}^{a \nu} W_{\nu}^{b \rho} W_{\rho}^{c \mu}
\end{array}
$$

where $f^{A B C}$ and $\varepsilon^{a b c}$ are the structure constants of colour $S U(3)$ and weak $S U(2)$, respectively. The dual field strength is defined by

$$
\tilde{X}_{\mu \nu}=\frac{1}{2} \varepsilon_{\mu \nu \rho \sigma} X^{\rho \sigma}, \quad \varepsilon^{0123}=-1
$$

Similar comments apply to the dimension- 5 operators of class $\psi^{2} U X$. Again there are only a few structures, which can be written down as follows:

$$
\begin{array}{cc}
\mathcal{O}_{\psi X 1,2}=g_{s} \bar{q} \sigma^{\mu \nu} G_{\mu \nu} U P_{ \pm} r & \\
\mathcal{O}_{\psi X 3,4}=g \bar{q} \sigma^{\mu \nu} W_{\mu \nu} U P_{ \pm} r, & \mathcal{O}_{\psi X 5}=g \bar{l} \sigma^{\mu \nu} W_{\mu \nu} U P_{-} \eta \\
\mathcal{O}_{\psi X 6,7}=g^{\prime} \bar{q} \sigma^{\mu \nu} B_{\mu \nu} U P_{ \pm} r, & \mathcal{O}_{\psi X 8}=g^{\prime} \bar{l} \sigma^{\mu \nu} B_{\mu \nu} U P_{-} \eta
\end{array}
$$




\subsection{Baryon and lepton number violating operators}

The suppression of $B$-violating operators has to be very strong in view of the stringent lower limit on the proton lifetime, pointing to the energy scale of grand unified theories (GUT), which could naturally generate such operators. Such a large scale is unrelated to the scale of electroweak symmetry breaking. We will therefore not include those terms explicitly in our basis. The five $B$ (and $L$ ) violating operators built from four Standard-Model fermions are well known [26] and can also be found in [24].

Similarly, $B$-conserving but $L$-violating operators can be considered. The fundamental scale of lepton-number violation is not well determined and might in principle range from a few $\mathrm{TeV}$ to values that are many orders of magnitude larger. Since $L$ violation is relevant for a general description of neutrino masses, we want to include the dominant effects of this type in the lowest-order Lagrangian. In our framework the leading $L$-violating operator has dimension 3 and can be written as

$$
Q_{L V}=l^{T} C U^{*} P_{+} U^{\dagger} l
$$

where $C=i \gamma^{2} \gamma^{0}$ is the charge-conjugation matrix. A similar operator has already been considered in [27]. $Q_{L V}$ is unique up to the various possible flavour assignments of $l$. This is in full analogy to the well-known $L$-violating dimension- 5 operator in the usual Standard Model

$$
\left(\tilde{\phi}^{\dagger} l\right)^{T} C \tilde{\phi}^{\dagger} l
$$

The operator in (42) is the equivalent of (43) in a theory without a light StandardModel Higgs boson. In the limit $U \rightarrow 1$, (42) reduces to $\nu_{L}^{T} C \nu_{L}$, the Majorana mass term of the left-handed neutrinos. Correspondingly, the very small neutrino masses set the scale for the coefficient of $Q_{L V}$ in the Lagrangian. The smallness of this coefficient can be understood in the standard way invoking the seesaw mechanism. In this scenario right-handed neutrinos with large Majorana masses can be included into the effective Lagrangian by adding the term

$$
\mathcal{L}_{\nu_{R}}=-M_{D} \bar{l} U P_{+} \eta-\frac{1}{2} M_{R} \nu_{R}^{T} C \nu_{R}+\text { h.c. }
$$

Integrating out $\nu_{R}$ leads to $Q_{L V}$ in (42). This consideration illustrates that a physical Higgs boson is not essential for generating neutrino masses of either Dirac or Majorana type.

Since the leading lepton-number violating operator in (42) has a very small coefficient, we will not list explicitly the formally next-to-leading order operators in the $L$-violating sector.

\section{$5 \quad$ Standard Model with a heavy Higgs boson}

In the following section we would like to illustrate how the nonlinear effective Lagrangian of electroweak interactions, including next-to-leading order terms, arises in the context 
of a specific model. For this purpose we consider the conventional Standard Model with a heavy Higgs boson of mass $m_{h} \sim \Lambda \gg v$, which is integrated out at scales below $m_{h}$. Although by itself the SM with a heavy Higgs particle is not a realistic description of Nature, it provides the simplest example of a renormalizable theory that reduces to the nonlinear effective Lagrangian at electroweak energies. A similar discussion in the context of chiral perturbation theory and the linear $\sigma$-model, applied to low-energy QCD, can be found for instance in [28].

The Higgs sector of the renormalizable Standard Model can be written as

$$
\begin{aligned}
\mathcal{L}_{H}= & \frac{1}{4}\left\langle D_{\mu} H^{\dagger} D^{\mu} H\right\rangle+\frac{\mu^{2}}{4}\left\langle H^{\dagger} H\right\rangle-\frac{\lambda}{16}\left\langle H^{\dagger} H\right\rangle^{2} \\
& -\left(\bar{q} Y_{u} H P_{+} r+\bar{q} Y_{d} H P_{-} r+\bar{l} Y_{e} H P_{-} \eta+\text { h.c. }\right)
\end{aligned}
$$

where the ordinary Higgs doublet, written in matrix notation $H=(\tilde{\phi}, \phi)$, has been parametrized as (see e.g. [29])

$$
H \equiv(v+h) U, \quad U^{\dagger} U=1
$$

Here $U$ is the $S U(2)$ matrix field defined in (4) and $h$ is the Higgs boson, which transforms as a singlet under the SM gauge group. With (46) the Lagrangian in (45) becomes

$$
\mathcal{L}_{H}=\frac{v^{2}}{4}\left\langle D_{\mu} U^{\dagger} D^{\mu} U\right\rangle-v\left(\bar{q} Y_{u} U P_{+} r+\ldots\right)+\mathcal{L}_{H, h}
$$

where

$$
\mathcal{L}_{H, h}=\frac{1}{2} h\left(-\partial^{2}-m_{h}^{2}\right) h-\frac{m_{h}^{2}}{2 v} h^{3}-\frac{m_{h}^{2}}{8 v^{2}} h^{4}+h J_{1}+\frac{h^{2}}{2} J_{2}
$$

Here we have used $m_{h}^{2}=2 \mu^{2}$ and $\lambda=\mu^{2} / v^{2}$, and the definitions

$$
J_{1}=\frac{v}{2}\left\langle D_{\mu} U^{\dagger} D^{\mu} U\right\rangle-\left(\bar{q} Y_{u} U P_{+} r+\ldots\right), \quad J_{2}=\frac{1}{2}\left\langle D_{\mu} U^{\dagger} D^{\mu} U\right\rangle
$$

Removing the heavy Higgs boson $h$ from the theory, $\mathcal{L}_{H}$ in $(47)$ reduces to the lowestorder electroweak chiral Lagrangian. Subleading terms in this Lagrangian are generated when corrections from virtual $h$ are taken into account. Keeping only the effects of single tree-level exchange of the heavy Higgs particle, the next-to-leading order terms read

$$
\begin{aligned}
& \mathcal{L}_{H, h}^{e f f}=\frac{J_{1}^{2}}{2 m_{h}^{2}}= \\
& \frac{v^{2}}{8 m_{h}^{2}}\left\langle D_{\mu} U^{\dagger} D^{\mu} U\right\rangle^{2}-\frac{v}{2 m_{h}^{2}}\left\langle D_{\mu} U^{\dagger} D^{\mu} U\right\rangle\left(\bar{q} Y_{u} U P_{+} r+\bar{q} Y_{d} U P_{-} r+\bar{l} Y_{e} U P_{-} \eta+\text { h.c. }\right) \\
& +\frac{1}{2 m_{h}^{2}}\left(\bar{q} Y_{u} U P_{+} r+\bar{q} Y_{d} U P_{-} r+\bar{l} Y_{e} U P_{-} \eta+\text { h.c. }\right)^{2}
\end{aligned}
$$

The Lagrangian $\mathcal{L}_{H, h}^{e f f}$ arises from the terms $-m_{h}^{2} h^{2} / 2+h J_{1}$ in (48) upon completing the square when integrating out $h$ in the path integral. Further terms generated by 
the exchange of $h$ bosons at tree level, such as $J_{1}^{2} J_{2}$ or $J_{1}^{3}$, require more than one $h$ propagator. They are therefore suppressed by additional powers of $1 / m_{h}^{2}$.

The next-to-leading operators in (50) are a subset of the complete basis listed in section 4. They illustrate how operators of different canonical dimension emerge at a given order of the effective Lagrangian. In particular, operators of dimension 4, 5 and 6 are present in (50). Specifically, the following terms out of the basis of NLO operators appear: The pure $U$-field operator $\mathcal{O}_{D 1}$, the fermion bilinear operators $\mathcal{O}_{\psi S 1}, \mathcal{O}_{\psi S 2}$, $\mathcal{O}_{\psi S 7}$, and their hermitian conjugates, as well as all 4-fermion operators coming from the square of the Yukawa terms. Up to hermitian conjugates, there are 12 such 4-fermion operators, which are given by

$$
\begin{aligned}
& \bar{q} U P_{+} r \bar{r} P_{+} U^{\dagger} q=-\frac{1}{4} \mathcal{O}_{L R 1}-\frac{1}{2} \mathcal{O}_{L R 10} \\
& \bar{q} U P_{-} r \bar{r} P_{-} U^{\dagger} q=-\frac{1}{4} \mathcal{O}_{L R 3}+\frac{1}{2} \mathcal{O}_{L R 12} \\
& \bar{l} U P_{-} \eta \bar{\eta} P_{-} U^{\dagger} l=-\frac{1}{4} \mathcal{O}_{L R 8}+\frac{1}{2} \mathcal{O}_{L R 17} \\
& \bar{q} U P_{-} r \bar{\eta} P_{-} U^{\dagger} l=-\frac{1}{4} \mathcal{O}_{L R 9}+\frac{1}{2} \mathcal{O}_{L R 18}
\end{aligned}
$$

together with

$$
\mathcal{O}_{S T 5}, \mathcal{O}_{S T 9}, \mathcal{O}_{F Y 1}, \mathcal{O}_{F Y 3}, \mathcal{O}_{F Y 5}, \mathcal{O}_{F Y 7}, \mathcal{O}_{F Y 9}, \mathcal{O}_{F Y 10}
$$

\section{Standard Model with a light Higgs boson}

In the conventional Standard Model with a physical Higgs field the next-to-leading order corrections to the renormalizable part are given by dimension- 6 operators in the effective Lagrangian (up to the single dimension-5 operator that generates Majorana masses for neutrinos given in (43)). The list of these operators has been compiled by Buchmüller and Wyler in [25]. It has recently been revised and updated in a new systematic study by Grzadkowski et al. [24]. In this section we show how the complete list of operators in [24] can be reproduced from the next-to-leading operators in our effective theory. We have checked the contents of [24] and fully agree with the results of this paper.

The matrix $H=(\tilde{\phi}, \phi)$, where $\phi$ is the linearly transforming Higgs field, transforms in the same way as the Goldstone field $U$. We may therefore replace $U \rightarrow H$ in the operators

of the nonlinear theory without affecting the required transformation properties, gauge invariance in particular. However, introducing the linear field $H$ implies two important differences with respect to the nonlinear version based on $U$. First, we no longer have the constraint $U^{\dagger} U=1$, so that factors of $\left\langle H^{\dagger} H\right\rangle$ can now appear as independent variables in the Lagrangian (note however that $\left\langle H^{\dagger} H T_{3}\right\rangle=0$ ). Second, since the lowest-order Lagrangian becomes renormalizable with the transition to the linear Higgs field, the newphysics scale $\Lambda$ may be taken to decouple from the electroweak scale, $v / \Lambda \rightarrow 0$. Therefore, 
the field $H$ enters the power counting with its canonical dimension $\operatorname{dim} H=1$. The ordering of terms in the effective Lagrangian is then governed by dimensional counting alone. For example, at lowest order, where $\mathcal{L}=\mathcal{L}_{4}+\mathcal{L}_{U}$ from (2) and (7), one has to substitute $U \rightarrow H / v$ and add the invariants $\left\langle H^{\dagger} H\right\rangle$ and $\left(\left\langle H^{\dagger} H\right\rangle\right)^{2}$ to recover all terms up to dimension 4 of the renormalizable Standard Model. Note that with the replacement $U \rightarrow H / v$ the custodial symmetry breaking term in (9) becomes an operator of dimension 6.

Applying this reasoning to the next-to-leading-order terms based on the nonlinear theory, we first have to include additional operators that involve the invariant $\left\langle H^{\dagger} H\right\rangle \sim$ $\phi^{\dagger} \phi$. These are easily enumerated at the dimension-6 level. The first possibility is the (unique) operator with six factors of $\phi,\left(\phi^{\dagger} \phi\right)^{3}$ (class $\phi^{6}$ in [24]). Next we have $\left(\phi^{\dagger} \phi\right)^{2}$ in combination with a dimension-2 operator, which can only be $D^{2}$. This gives the operator $\left(\phi^{\dagger} \phi\right) \partial^{2}\left(\phi^{\dagger} \phi\right)$ (class $\phi^{4} D^{2}$ in [24]), which is unique up to total derivatives. Finally, one may write down the operators with a single factor of $\phi^{\dagger} \phi$, multiplied by any gauge invariant operator of dimension 4. The candidates for the latter are just the terms in the leading-order renormalizable Lagrangian. This gives immediately the three operators

$$
\phi^{\dagger} \phi \bar{l} e \phi, \quad \phi^{\dagger} \phi \bar{q} d \phi, \quad \phi^{\dagger} \phi \bar{q} u \tilde{\phi},
$$

corresponding to the Yukawa terms (class $\psi^{2} \phi^{3}$ in [24]). Similarly one has $\phi^{\dagger} \phi X_{\mu \nu} X^{\mu \nu}$ and $\phi^{\dagger} \phi X_{\mu \nu} \tilde{X}^{\mu \nu}$ for the gauge fields $X=G, W, B$ (class $X^{2} \phi^{2}$ in [24]). No new operators arise from the Higgs-potential terms $m^{2} \phi^{\dagger} \phi$ and $\left(\phi^{\dagger} \phi\right)^{2}$, nor from the fermion kinetic terms $\bar{\psi} i \not D \psi$, where the leading-order equations of motion can be applied to reduce $\phi^{\dagger} \phi \bar{\psi} i \not D \psi$ to structures already listed above. For the only remaining possibility we have, using equations of motion and integration by parts, [24]

$$
\phi^{\dagger} \phi\left(D_{\mu} \phi\right)^{\dagger} D^{\mu} \phi=\frac{1}{2} \phi^{\dagger} \phi \partial^{2}\left(\phi^{\dagger} \phi\right)+\ldots
$$

The operator on the right is the term in class $\phi^{4} D^{2}$ mentioned before and the ellipsis denotes total derivatives and further operators already encountered above. If the Higgs boson kinetic term is written differently, we would have $\phi^{\dagger} \phi \phi^{\dagger} D^{2} \phi$, which is again not independent upon using the equations of motion. In total, therefore, one finds 11 operators involving factors of $\phi^{\dagger} \phi$.

Next, there are the operators of dimension 6 that do not include any factor of $U$ and which are thus identical in the nonlinear and in the linear theory. These are the 4-fermion operators without $U$ fields $\left(\psi^{4}\right)$ and the operators with three factors of fieldstrength tensors $\left(X^{3}\right)$.

A further class of operators are the terms in the nonlinear theory that become operators of dimension larger than 6 upon replacing $U \rightarrow H$. They are therefore absent in the linear Higgs theory at order $1 / \Lambda^{2}$. The operators of this type are those in our classes $U D^{4}, \psi^{2} U D^{2}$ and $\psi^{4} U$.

This leaves the cases $X^{2} U, X U D^{2}, \psi^{2} U D$ and $\psi^{2} U X$ of next-to-leading operators in the nonlinear theory, as well as the operator in (9). They translate into dimension-6 operators of the Standard Model when $U \rightarrow H=(\tilde{\phi}, \phi)$, as described in the following. 
The custodial-symmetry breaking operator in (9) becomes, up to a total divergence,

$$
\left(\left\langle U^{\dagger} D_{\mu} U T_{3}\right\rangle\right)^{2} \rightarrow-\frac{1}{4}\left(\phi^{\dagger} \phi\right) \partial^{2}\left(\phi^{\dagger} \phi\right)-\left(D_{\mu} \phi^{\dagger} \phi\right)\left(\phi^{\dagger} D^{\mu} \phi\right)
$$

The first term on the right has already been listed above. The second term is the remaining operator of class $\phi^{4} D^{2}$ in [24].

Inspection of (21) and (22) shows that most of the operators in our classes $X^{2} U$ and $X U D^{2}$ translate into operators of dimension higher than 6 . The exceptions are

$$
B_{\mu \nu}\left\langle U^{\dagger} W^{\mu \nu} U T_{3}\right\rangle \rightarrow-B_{\mu \nu} \phi^{\dagger} W^{\mu \nu} \phi
$$

and the analogous term where $W_{\mu \nu} \rightarrow \tilde{W}_{\mu \nu}$. These two operators complete class $X^{2} \phi^{2}$ in [24].

Among the operators of class $\psi^{2} U D$ in (25), some contain four factors of $U$-fields and become operators of dimension 8 when we replace $U \rightarrow H$. The remaining structures reduce to the eight dimension- 6 operators in class $\psi^{2} \phi^{2} D$ of [24]:

$$
\begin{gathered}
\bar{\psi} \gamma^{\mu} \psi\left\langle U^{\dagger} i D_{\mu} U T_{3}\right\rangle \rightarrow-\frac{1}{2} \bar{\psi} \gamma^{\mu} \psi\left(\phi^{\dagger} i D_{\mu} \phi-i D_{\mu} \phi^{\dagger} \phi\right), \quad \psi=q, u, d, l, e \\
\bar{\psi} \gamma^{\mu} i D_{\mu} U U^{\dagger} \psi \rightarrow 2 \bar{\psi} \gamma^{\mu} T^{a} \psi\left(\phi^{\dagger} T^{a} i D_{\mu} \phi-i D_{\mu} \phi^{\dagger} T^{a} \phi\right), \quad \psi=q, l \\
\bar{u} \gamma^{\mu} d\left\langle U^{\dagger} i D_{\mu} U P_{21}\right\rangle \rightarrow \bar{u} \gamma^{\mu} d i \tilde{\phi}^{\dagger} D_{\mu} \phi
\end{gathered}
$$

Finally, since

$$
U P_{+} r \rightarrow \tilde{\phi} u, \quad U P_{-} r \rightarrow \phi d, \quad U P_{-} \eta \rightarrow \phi e
$$

the eight operators in our class $\psi^{2} U X$ (41) reduce to the operators in class $\psi^{2} X \phi$ of [24]. This completes the demonstration of how the full basis of dimension- 6 operators given in [24] can be recovered from the next-to-leading order terms in the nonlinear Higgs model.

\section{Example of renormalization at NLO}

A comprehensive discussion of renormalization of the effective theory is beyond the scope of the present paper. We limit ourselves to a particular example, which should serve to illustrate the essential features of renormalization at next-to-leading order in the electroweak chiral Lagrangian.

Consider the amplitude for $t_{R} t_{R} \rightarrow t_{L} t_{L}$ scattering, taking the gaugeless limit for simplicity. The leading-order ( $\mathrm{LO}$ ) amplitude is given by the tree-level contributions from the LO Lagrangian in (2) and (7). It arises from the exchange of a neutral Goldstone boson and reads

$$
A_{0}=i y^{2}\left(\frac{\tau_{t}}{t}-\frac{\tau_{u}}{u}\right)
$$


where $y$ is the top-quark Yukawa coupling, $t=\left(p_{1}-p_{3}\right)^{2}, u=\left(p_{1}-p_{4}\right)^{2}$ are Mandelstam variables and the fermion spinors are denoted by

$$
\tau_{t} \equiv \bar{t}_{L}\left(p_{3}\right) t_{R}\left(p_{1}\right) \bar{t}_{L}\left(p_{4}\right) t_{R}\left(p_{2}\right), \quad \tau_{u} \equiv \bar{t}_{L}\left(p_{4}\right) t_{R}\left(p_{1}\right) \bar{t}_{L}\left(p_{3}\right) t_{R}\left(p_{2}\right)
$$

The NLO amplitude arises both from one-loop corrections based on the LO Lagrangian, and from tree-level contributions of the NLO Lagrangian. The Goldstone-boson loop diagram shown in Fig. 1 gives the correction

$$
A_{1, \text { loop }}=\frac{i}{16 \pi^{2}} \frac{y^{2}}{v^{2}} \frac{3}{2}\left[\tau_{t}\left(\ln \frac{\mu^{2}}{-t}+\frac{1}{\epsilon}-\gamma+\ln 4 \pi+2\right)-(t \rightarrow u)\right]
$$

This contribution is divergent. It needs to be renormalized by the appropriate counterterm, which is provided by the following piece of the NLO Lagrangian (see eq. (37))

$$
\Delta \mathcal{L}=\frac{c}{\Lambda^{2}} \mathcal{O}_{F Y 1} \rightarrow \frac{c}{16 \pi^{2} v^{2}} \bar{t}_{L} t_{R} \bar{t}_{L} t_{R}
$$

where the last term gives the part of the operator that contributes to the considered amplitude at tree level. This contribution is

$$
A_{1, \text { tree }}=\frac{2 i c^{(0)}}{16 \pi^{2} v^{2}}\left(\tau_{t}-\tau_{u}\right)
$$

with the bare coefficient $c^{(0)}$. Absorbing the divergence in (61) into this coefficient by minimal subtraction (and dropping a constant) one finds for the renormalized NLO contribution

$$
A_{1}=A_{1, \text { loop }}+A_{1, \text { tree }}=\frac{i}{16 \pi^{2}} \frac{y^{2}}{v^{2}} \frac{3}{2}\left[\tau_{t}\left(\ln \frac{\mu^{2}}{-t}+\frac{4}{3 y^{2}} c(\mu)\right)-(t \rightarrow u)\right]
$$

The NLO term $A_{1}$ is suppressed with respect to the LO amplitude $A_{0}$ as $p^{2} / \Lambda^{2}$, where $p \sim v$ is the typical momentum scale of the process. $A_{1}$ depends on $c(\mu)$, a free parameter of the effective theory, which cancels the dependence on the arbitrary scale $\mu$. Independently of the local term provided by $c, A_{1}$ reproduces the correct logarithmic dependence of the amplitude on $t$ and $u$ at NLO.

It is interesting to compare the effective-theory calculation just described with the analogous calculation in the usual Standard Model that includes a heavy Higgs boson. The latter model can be viewed as the simplest UV completion of the electroweak chiral Lagrangian. The LO amplitude for $t_{R} t_{R} \rightarrow t_{L} t_{L}$ in the gaugeless limit coincides with the result in the effective theory given in (59). At NLO, that is, at order $p^{2} / \Lambda^{2}$, the amplitude receives a contribution from tree-level Higgs exchange

$$
A_{1, \text { tree }}^{h}=i \frac{y^{2}}{m_{h}^{2}}\left(\tau_{t}-\tau_{u}\right)
$$


where the Higgs-boson mass $m_{h}$ is assumed to be large, $p^{2} \ll m_{h}^{2} \lesssim \Lambda^{2}=16 \pi^{2} v^{2}$. Including 1-loop corrections, one obtains the following term with (large) logarithms of $\mathcal{O}\left(\ln m_{h}^{2} / p^{2}\right)$

$$
A_{1, \text { loop }}^{h}=\frac{i}{16 \pi^{2}} \frac{y^{2}}{v^{2}} \frac{3}{2}\left[\tau_{t} \ln \frac{m_{h}^{2}}{-t}-(t \rightarrow u)\right]
$$

This term comes from the Higgs-exchange diagram with a Goldstone-boson loop as a self-energy insertion in the Higgs propagator. Other loop corrections do not give rise to a large logarithm and in (66) we have neglected all such nonlogarithmic terms. Combining (65) and (66), the NLO correction in the heavy-Higgs model reads

$$
A_{1}^{h}=A_{1, \text { tree }}^{h}+A_{1, \text { loop }}^{h}=\frac{i}{16 \pi^{2}} \frac{y^{2}}{v^{2}} \frac{3}{2}\left[\tau_{t}\left(\ln \frac{m_{h}^{2}}{-t}+\frac{2}{3} \frac{16 \pi^{2} v^{2}}{m_{h}^{2}}\right)-(t \rightarrow u)\right]
$$

If the Higgs boson is moderately heavy, $p^{2} \ll m_{h}^{2} \ll \Lambda^{2}$, the Higgs sector is still perturbative. In this case, the tree-level term $\sim v^{2} / m_{h}^{2}$ in (67) is dominating over the logarithmic piece. As $m_{h}$ increases towards $\Lambda$, the Higgs sector becomes strongly coupled and (67) ceases to be reliable. Besides, the tree-level term starts being comparable to order-one contributions we have neglected in (67). In this situation we might still consider (67) as a rough estimate of the full amplitude. In any case, (67) defines a particular UV completion of the effective-theory amplitude (64). Within such a UV completion, the coefficient $c$ in (64) can be determined. In the present example one finds, equating (64) and (67),

$$
c(\mu)=y^{2}\left(\frac{3}{4} \ln \frac{m_{h}^{2}}{\mu^{2}}+\frac{8 \pi^{2} v^{2}}{m_{h}^{2}}\right)
$$

The logarithmic piece can be fixed from the one-loop UV divergence of the effective theory, without knowing the details of the physics at the high-energy scale $\Lambda$. However, as the example illustrates especially for a moderate $m_{h}$, the nonlogarithmic, constant term may be sizable. In this case the leading logarithmic approximation will not be accurate. Similar considerations have been given in [9].

\section{Conclusions}

In this article we have studied an effective field theory (EFT) description of the Standard Model assuming that the mechanism for spontaneous electroweak symmetry breaking is triggered by strong dynamics at the $\mathrm{TeV}$ scale. The resulting theory contains the fields of the Standard Model but, instead of describing electroweak symmetry breaking by introducing a Higgs doublet (linear sigma model), one uses a nonlinear realization for the Goldstone modes.

This article focusses mainly on clarifying the systematics of such an approach: in particular, we discuss in detail the power-counting of the $v / \Lambda$ expansion, providing the formulae needed to determine the complete list of NLO operators. This list is presented in Landau gauge and, for phenomenological convenience, also in unitary gauge. At NLO 
there are 11 operators without fermion fields, 25 operators involving fermion bilinears and 64 four-quark operators. It is worth recalling that not all of the previous operators are required as counterterms of our effective field theory. While in some cases it is relatively easy to identify them, a general and systematic identification of the counterterms would require to carry out the full renormalization program for the LO operators, something that lies beyond the scope of the present article.

Several checks have been performed to eliminate redundancies between operators. In particular, we have emphasized relations between operators from the use of integration by parts and the equations of motion $[17,18]$. These relations affect the operators contributing to triple-gauge-boson interactions.

As an additional cross-check we have reexpressed our basis of operators in the linear representation. This particular case corresponds to deriving the NLO (dimension-6) operators in the Standard Model with a Higgs boson. Our results independently confirm the recent analysis of [24]. The sets of NLO operators in our scenario and in the presence of a Higgs boson are seen to be related, but there are systematic differences. This is an additional motivation to study the phenomenology associated with our EFT basis of operators, especially in electroweak precision observables but also in top-quark physics.

Finally, it is worth stressing that the approach undertaken in this article assumes the minimal content of Standard Model fields: the electroweak symmetry breaking sector is characterized only by three Goldstone bosons, which are the longitudinal modes of the $W$ and $Z$ gauge bosons. Therefore, no extraneous particles are introduced. However, further light, electroweak-scale particles might in principle exist. It would be interesting to study the systematics of a similar EFT scenario under this generalization.

\section{A Derivation of the power-counting formula}

To obtain the power-counting formula in (15) we start from the different types of vertices contained in the leading order Lagrangian (2) and (7). Denoting left-handed (righthanded) fermions, Goldstone bosons and gauge fields by $\psi_{L}\left(\psi_{R}\right), \varphi$ and $X_{\mu}$, respectively, these vertices have the schematic form of $\varphi^{2 i}$ (2i Goldstone boson interaction), $\bar{\psi}_{L(R)} \psi_{R(L)} \varphi^{k}$ (Yukawa interaction), $X_{\mu} \varphi^{l}, X_{\mu}^{2} \varphi^{s}$ (gauge-Goldstone boson interaction), $X_{\mu}^{4}, X_{\mu}^{3}$ (gauge boson self interaction), and $\bar{\psi}_{L(R)} \psi_{L(R)} X_{\mu}$ (fermion-gauge boson coupling). For each vertex, the factors of momentum $p$, Yukawa coupling $y$, gauge coupling $g$ and electroweak scale $v$, contributing to the power counting in any diagram, are given by:

\begin{tabular}{c|c|c|c|c|c|c}
$\varphi^{2 i}$ & $\bar{\psi}_{L(R)} \psi_{R(L)} \varphi^{k}$ & $X_{\mu} \varphi^{l}$ & $X_{\mu}^{2} \varphi^{s}$ & $X_{\mu}^{4}$ & $X_{\mu}^{3}$ & $\bar{\psi}_{L(R)} \psi_{L(R)} X_{\mu}$ \\
\hline \hline$p^{2} / v^{2 i-2}$ & $y / v^{k-1}$ & $g p / v^{l-1}$ & $g^{2} / v^{s-2}$ & $g^{2}$ & $g p$ & $g$ \\
\hline$n_{i}$ & $\nu_{k}$ & $m_{l}$ & $r_{s}$ & $x$ & $u$ & $z_{L}\left(z_{R}\right)$
\end{tabular}

Here the last line defines the number of the corresponding vertices in a given diagram. A diagram $\mathcal{D}$ with $L$ loops, $\mathcal{F}_{L}\left(\mathcal{F}_{R}\right), \mathcal{B}$, and $\mathcal{V}$, fermion, Goldstone, and gauge field propagators, $F_{L}\left(F_{R}\right), B$, and $V$ external fermion, Goldstone, and gauge field lines, and 
with the numbers of vertices introduced in (69), contains the factors $\left(z \equiv z_{L}+z_{R}\right)$

$$
\begin{aligned}
\mathcal{D} \sim & \frac{v^{2 L}}{\Lambda^{2 L}} \frac{y^{\sum_{k} \nu_{k}} g^{\sum_{l} m_{l}+\sum_{s} 2 r_{s}+2 x+u+z}}{v^{\sum_{i}(2 i-2) n_{i}+\sum_{k}(k-1) \nu_{k}+\sum_{l}(l-2) m_{l}+\sum_{s}(s-2) r_{s}}} \\
& \times p^{4 L+\sum_{i} 2 n_{i}+\sum_{l} m_{l}+u-2 \mathcal{B}-2 \mathcal{V}-\mathcal{F}_{L}-\mathcal{F}_{R}-V} \bar{\psi}_{L}^{F_{L}^{1}} \psi_{L}^{F_{L}^{2}} \bar{\psi}_{R}^{F_{R}^{1}} \psi_{R}^{F_{R}^{2}} \varphi^{B}\left(X_{\mu \nu}\right)^{V}
\end{aligned}
$$

In writing (70) we have made explicit the external fermion, Goldstone-boson and gaugeboson fields. We have written the gauge field in terms of the field strength $X_{\mu \nu}$ (rather than the gauge potential $X_{\mu}$ ) and correspondingly associated a factor of $p$ with each gauge field, $X_{\mu \nu} \sim p X_{\mu}$. This is because we are interested in the power counting for gaugeinvariant operators containing factors of field-strength tensors. Operators with factors of $X_{\mu}$, but without associated momentum $p$, are not gauge-invariant by themselves and are related to operators with (gauge-covariant) derivatives. The first factor in $(70)$ is the loop factor $1 /\left(16 \pi^{2}\right)^{L}$, where we have identified $1 /\left(16 \pi^{2}\right)=v^{2} / \Lambda^{2}$. The remaining factors follow immediately from collecting all parts of the diagram.

The expression (70) can be put into a more useful form by employing the well-known topological identities for Feynman graphs

$$
\begin{gathered}
F_{L}+2 \mathcal{F}_{L}=\sum_{k} \nu_{k}+2 z_{L}, \quad F_{R}+2 \mathcal{F}_{R}=\sum_{k} \nu_{k}+2 z_{R} \\
B+2 \mathcal{B}=\sum_{i} 2 i n_{i}+\sum_{k} k \nu_{k}+\sum_{l} l m_{l}+\sum_{s} s r_{s} \\
V+2 \mathcal{V}=\sum_{l} m_{l}+\sum_{s} 2 r_{s}+4 x+3 u+z \\
L=\mathcal{F}_{L}+\mathcal{F}_{R}+\mathcal{B}+\mathcal{V}-\sum_{i} n_{i}-\sum_{k} \nu_{k}-\sum_{l} m_{l}-\sum_{s} r_{s}-x-u-z+1
\end{gathered}
$$

Using these five equations to eliminate the five quantities $\mathcal{F}_{L}, \mathcal{F}_{R}, \mathcal{B}, \mathcal{V}$ and $L$, one finds for the power $d$ of momentum factor $p$ in (70)

$$
d=4-B-2 V-\frac{3}{2} F_{L}-\frac{3}{2} F_{R}+\sum_{i}(2 i-2) n_{i}+\sum_{k}(k-1) \nu_{k}+\sum_{l}(l-2) m_{l}+\sum_{s}(s-2) r_{s}
$$

This corresponds to the standard expression for the superficial degree of divergence of a diagram, where the nonrenormalizable interactions are seen to give positive contributions that increase with the number of vertices. This is a correct result, but still not immediately useful for our purposes. To proceed, we may eliminate the positive contributions of the nonrenormalizable terms with the help of

$$
2 L=2-B-V-F_{L}-F_{R}+\sum_{i}(2 i-2) n_{i}+\sum_{k} k \nu_{k}+\sum_{l}(l-1) m_{l}+\sum_{s} s r_{s}+2 x+u+z
$$


which follows from (74) after eliminating $\mathcal{F}_{L}, \mathcal{F}_{R}, \mathcal{B}, \mathcal{V}$. Taking the difference between (75) and (76), the positive vertex terms in (75) cancel, and one has

$$
d=2 L+2-\frac{F_{L}+F_{R}}{2}-V-\sum_{k} \nu_{k}-\sum_{l} m_{l}-\sum_{s} 2 r_{s}-2 x-u-z
$$

Using (77) and (76) in (70) we finally obtain (15).

\section{B Technical aspects of operator building}

\section{B.1 Operators without fermions}

For this subset of operators the most convenient method is to list the elementary building blocks of $S U(2)$ algebra, as was done in [9]. With $S U(2)$ elements, only traces of at most 3 elements are not redundant. This follows from

$$
T^{a} T^{b}=\frac{1}{4} \delta^{a b}+\frac{i}{2} \varepsilon^{a b c} T^{c}
$$

which implies $\left\langle T^{a} T^{b}\right\rangle \sim \delta^{a b}$ and $\left\langle T^{a} T^{b} T^{c}\right\rangle \sim \varepsilon^{a b c}$. For our present purposes we will only need as $S U(2)$ elements the chiral vector, $L_{\mu}=U D_{\mu} U^{\dagger}, W_{\mu \nu}$ and the scalar spurion $\tau_{L}=U T_{3} U^{\dagger}$, all of which are (anti-) hermitean and traceless. That singles out the following elementary building blocks:

$$
\begin{array}{lr}
\left\langle L_{\mu} L_{\nu}\right\rangle, & \left\langle W_{\mu \nu} L_{\lambda}\right\rangle, \\
\left\langle\tau_{L} L_{\mu}\right\rangle, & \left\langle\tau_{L} W_{\mu \nu}\right\rangle, \\
\left\langle L_{\mu} L_{\nu} L_{\lambda}\right\rangle, & \left\langle W_{\mu \nu} L_{\lambda} L_{\rho}\right\rangle, \\
\left\langle\tau_{L} L_{\mu} L_{\nu}\right\rangle, & \left\langle\tau_{L} W_{\mu \nu} L_{\lambda}\right\rangle
\end{array}
$$

The previous building blocks have to be assembled into the dimension-4 operators of classes $U D^{4}, X^{2} U$ and $X U D^{2}$, such that traces of 3 elements only show up once in any operator (since the product of two Levi-Civita tensors is reducible). Lorentz invariance then naturally selects the possible combinations. It is convenient to separate the possible operators into two categories: those with and without gauge field strengths. To NLO the former set consists of

$$
\begin{array}{lr}
\left\langle L_{\mu} L_{\nu}\right\rangle\left\langle L^{\mu} L^{\nu}\right\rangle, & \left\langle L_{\mu} L^{\mu}\right\rangle\left\langle L_{\nu} L^{\nu}\right\rangle, \\
\left\langle L_{\nu} L^{\nu}\right\rangle\left\langle\tau_{L} L_{\mu}\right\rangle^{2}, & \left\langle L_{\mu} L_{\nu}\right\rangle\left\langle\tau_{L} L^{\mu}\right\rangle\left\langle\tau_{L} L^{\nu}\right\rangle, \\
\left\langle\tau_{L} L_{\mu}\right\rangle^{2}\left\langle\tau_{L} L_{\nu}\right\rangle^{2} &
\end{array}
$$

The list consists only of CP-conserving operators: operators involving purely Goldstone fields are symmetric under the exchange of at least two Lorentz indices and therefore cancel identically when contracted with the Levi-Civita symbol $\varepsilon_{\mu \nu \lambda \rho}$. 
In contrast, operators with gauge field strengths (at NLO) have contributions from $\mathrm{CP}$-conserving and $\mathrm{CP}$-violating sectors. We will discuss the CP-conserving sector in detail and later on generalize to the $\mathrm{CP}$-violating sector. The set of CP-conserving operators one can construct with the building blocks of Eq. (79) is

$$
\begin{aligned}
& \mathcal{O}_{X U 1}=g^{\prime} g B_{\mu \nu}\left\langle W^{\mu \nu} \tau_{L}\right\rangle \\
& \mathcal{O}_{X U 2}=g^{2}\left\langle W_{\mu \nu} \tau_{L}\right\rangle^{2} \\
& \mathcal{O}_{X U 3}=g \varepsilon_{\mu \nu \lambda \rho}\left\langle W^{\mu \nu} L^{\lambda}\right\rangle\left\langle\tau_{L} L^{\rho}\right\rangle \\
& \mathcal{O}_{X U 7}=i g^{\prime} B_{\mu \nu}\left\langle\tau_{L}\left[L^{\mu}, L^{\nu}\right]\right\rangle \\
& \mathcal{O}_{X U 8}=i g\left\langle W_{\mu \nu}\left[L^{\mu}, L^{\nu}\right]\right\rangle \\
& \mathcal{O}_{X U 9}=i g\left\langle W_{\mu \nu} \tau_{L}\right\rangle\left\langle\tau_{L}\left[L^{\mu}, L^{\nu}\right]\right\rangle
\end{aligned}
$$

One further operator that can be built in this way is not independent:

$$
\left\langle\left[\tau_{L}, L^{\mu}\right] W_{\mu \nu}\right\rangle\left\langle\tau_{L} L^{\nu}\right\rangle=\frac{1}{2}\left\langle\tau_{L} W_{\mu \nu}\right\rangle\left\langle\tau_{L}\left[L^{\mu}, L^{\nu}\right]\right\rangle-\frac{1}{4}\left\langle W_{\mu \nu}\left[L^{\mu}, L^{\nu}\right]\right\rangle
$$

To demonstrate this, we start from the identity $\left(\tau_{L}^{a} \equiv U T^{a} U^{\dagger}\right)$

$$
\left\langle\tau_{L} L_{\mu} W^{\mu \nu}\right\rangle\left\langle\tau_{L} L_{\nu}\right\rangle=\left\langle\tau_{L}^{a} L_{\mu} W^{\mu \nu}\right\rangle\left\langle\tau_{L}^{a} L_{\nu}\right\rangle-\left\langle\tau_{L}^{i} L_{\mu} W^{\mu \nu}\right\rangle\left\langle\tau_{L}^{i} L_{\nu}\right\rangle \quad(i \neq 3)
$$

However,

$$
\begin{aligned}
\left\langle\tau_{L}^{i} L_{\mu} W^{\mu \nu}\right\rangle\left\langle\tau_{L}^{i} L_{\nu}\right\rangle= & \frac{i}{8} \varepsilon^{i j k}\left(U^{\dagger} L_{\nu} U\right)^{i}\left(U^{\dagger} L_{\mu} U\right)^{j}\left(U^{\dagger} W^{\mu \nu} U\right)^{k} \\
= & \frac{i}{8} \varepsilon^{i 3 k}\left(U^{\dagger} L_{\nu} U\right)^{i}\left(U^{\dagger} L_{\mu} U\right)^{3}\left(U^{\dagger} W^{\mu \nu} U\right)^{k} \\
& +\frac{i}{8} \varepsilon^{i j 3}\left(U^{\dagger} L_{\nu} U\right)^{i}\left(U^{\dagger} L_{\mu} U\right)^{j}\left(U^{\dagger} W^{\mu \nu} U\right)^{3} \\
= & -\left\langle\tau_{L} L_{\nu} W^{\mu \nu}\right\rangle\left\langle L_{\mu} \tau_{L}\right\rangle+\left\langle L_{\nu} L_{\mu} \tau_{L}\right\rangle\left\langle W^{\mu \nu} \tau_{L}\right\rangle
\end{aligned}
$$

The terms with $\tau_{L}^{a}$ can be simplified with the $S U(2)$ relation $\left(\tau_{L}^{a}\right)_{i j}\left(\tau_{L}^{a}\right)_{k l}=\frac{1}{2} \delta_{i l} \delta_{j k}-\frac{1}{4} \delta_{i j} \delta_{k l}$ and one finally obtains (82).

Contrary to the list in (80), three of the operators in (81) can be shown to be redundant $[17,18]$. By using the identities

$$
\begin{aligned}
D_{\mu} \tau_{L} & =\left[\tau_{L}, L_{\mu}\right], \\
D_{\mu} L_{\nu}-D_{\nu} L_{\mu} & =-i g W_{\mu \nu}+i g^{\prime} B_{\mu \nu} \tau_{L}-\left[L_{\mu}, L_{\nu}\right],
\end{aligned}
$$

integration by parts and the equations of motion for the gauge fields,

$$
\begin{aligned}
\partial^{\mu} B_{\mu \nu} & =g^{\prime}\left[Y_{\psi} \bar{\psi} \gamma_{\nu} \psi+\frac{i}{2} v^{2}\left\langle\tau_{L} L_{\nu}\right\rangle\right] \\
D^{\mu} W_{\mu \nu}^{a} & =g\left[\bar{\psi}_{L} \gamma_{\nu} T^{a} \psi_{L}-\frac{i}{2} v^{2}\left\langle T^{a} L_{\nu}\right\rangle\right]
\end{aligned}
$$


one finds that

$$
\begin{aligned}
B_{\mu \nu}\left\langle\tau_{L}\left[L^{\mu}, L^{\nu}\right]\right\rangle & =-2 g^{\prime} Y_{\psi} \bar{\psi} \gamma_{\mu} \psi\left\langle\tau_{L} L^{\mu}\right\rangle-i g^{\prime} v^{2}\left\langle\tau_{L} L\right\rangle^{2}+i g B^{\mu \nu}\left\langle\tau_{L} W_{\mu \nu}\right\rangle-\frac{i g^{\prime}}{2} B_{\mu \nu} B^{\mu \nu} \\
\left\langle W_{\mu \nu}\left[L^{\mu}, L^{\nu}\right]\right\rangle= & g \bar{\psi}_{L} \gamma_{\mu} L^{\mu} \psi_{L}-\frac{i g}{2} v^{2}\left\langle L_{\mu} L^{\mu}\right\rangle+i g^{\prime} B^{\mu \nu}\left\langle\tau_{L} W_{\mu \nu}\right\rangle-i g\left\langle W_{\mu \nu} W^{\mu \nu}\right\rangle \\
\left\langle\tau_{L} W_{\mu \nu}\right\rangle\left\langle\tau_{L}\left[L^{\mu}, L^{\nu}\right]\right\rangle= & -\frac{g}{2} \bar{\psi}_{L} \gamma_{\mu} \tau_{L} \psi_{L}\left\langle\tau_{L} L^{\mu}\right\rangle+\frac{i g}{4} v^{2}\left\langle\tau_{L} L_{\mu}\right\rangle^{2}+\frac{i g}{2}\left\langle\tau_{L} W_{\mu \nu}\right\rangle^{2} \\
& +\frac{g}{4} \bar{\psi}_{L} \gamma_{\mu} L^{\mu} \psi_{L}-\frac{i g}{8} v^{2}\left\langle L_{\mu} L^{\mu}\right\rangle-\frac{i g}{4}\left\langle W_{\mu \nu} W^{\mu \nu}\right\rangle
\end{aligned}
$$

and thus $\mathcal{O}_{X U 7} \mathcal{O}_{X U 8}$ and $\mathcal{O}_{X U 9}$ above can be eliminated.

For the CP-violating sector one can likewise write:

$$
\begin{aligned}
\mathcal{O}_{X U 4} & =g^{\prime} g \varepsilon_{\mu \nu \lambda \rho}\left\langle\tau_{L} W^{\mu \nu}\right\rangle B^{\lambda \rho} \\
\mathcal{O}_{X U 5} & =g^{2} \varepsilon_{\mu \nu \lambda \rho}\left\langle\tau_{L} W^{\mu \nu}\right\rangle\left\langle\tau_{L} W^{\lambda \rho}\right\rangle \\
\mathcal{O}_{X U 6} & =g\left\langle W_{\mu \nu} L^{\mu}\right\rangle\left\langle\tau_{L} L^{\nu}\right\rangle \\
\mathcal{O}_{X U 10} & =i g^{\prime} \varepsilon_{\mu \nu \lambda \rho} B^{\mu \nu}\left\langle\tau_{L}\left[L^{\lambda}, L^{\rho}\right]\right\rangle \\
\mathcal{O}_{X U 11} & =i g \varepsilon_{\mu \nu \lambda \rho}\left\langle W^{\mu \nu}\left[L^{\lambda}, L^{\rho}\right]\right\rangle \\
\mathcal{O}_{X U 12} & =i g \varepsilon_{\mu \nu \lambda \rho}\left\langle W^{\mu \nu} \tau_{L}\right\rangle\left\langle\tau_{L}\left[L^{\lambda}, L^{\rho}\right]\right\rangle
\end{aligned}
$$

Similarly to the list in (81), there is no further independent structure beyond the six operators shown. This is because (82) also holds when $W_{\mu \nu} \rightarrow \tilde{W}_{\mu \nu}$. Proceeding in the same way as for the $\mathrm{CP}$-conserving sector, and recalling the Bianchi identity $\varepsilon_{\mu \nu \lambda \rho} \partial^{\mu} B^{\nu \lambda}=0=\varepsilon_{\mu \nu \lambda \rho} D^{\mu} W^{\nu \lambda}$, one can show the relations

$$
\begin{aligned}
\varepsilon_{\mu \nu \lambda \rho} B^{\mu \nu}\left\langle\tau_{L}\left[L^{\lambda}, L^{\rho}\right]\right\rangle & =i g \varepsilon_{\mu \nu \lambda \rho} B^{\mu \nu}\left\langle\tau_{L} W^{\lambda \rho}\right\rangle \\
\varepsilon_{\mu \nu \lambda \rho}\left\langle W^{\mu \nu}\left[L^{\lambda}, L^{\rho}\right]\right\rangle & =i g^{\prime} \varepsilon_{\mu \nu \lambda \rho} B^{\mu \nu}\left\langle\tau_{L} W^{\lambda \rho}\right\rangle \\
\varepsilon_{\mu \nu \lambda \rho}\left\langle\tau_{L} W^{\mu \nu}\right\rangle\left\langle\tau_{L}\left[L^{\lambda}, L^{\rho}\right]\right\rangle & =\frac{i g}{2} \varepsilon_{\mu \nu \lambda \rho}\left\langle\tau_{L} W^{\mu \nu}\right\rangle\left\langle\tau_{L} W^{\lambda \rho}\right\rangle
\end{aligned}
$$

and therefore $\mathcal{O}_{X U 10} \mathcal{O}_{X U 11}$ and $\mathcal{O}_{X U 12}$ above are redundant. The previous relations hold up to total derivatives, i.e., also neglecting the anomalous operator $\tilde{W}_{\mu \nu} W^{\mu \nu}$.

\section{B.2 Four-fermion operators}

The $(\bar{L} L)(\bar{L} L),(\bar{R} R)(\bar{R} R)$ and $(\bar{L} L)(\bar{R} R)$ groups are rather straightforward to dress with chiral fields. In order to study the groups $(\bar{L} R)(\bar{L} R)$ and $(\bar{L} R)(\bar{R} L)$ it is convenient to parametrize the chiral field as follows:

$$
U=\left(\begin{array}{cc}
e^{i \alpha} \cos \theta & -e^{i \beta} \sin \theta \\
e^{-i \beta} \sin \theta & e^{-i \alpha} \cos \theta
\end{array}\right) \equiv(\tilde{\omega}, \omega)
$$


The $S U(2)$ doublets trivially satisfy $\omega^{\dagger} \omega=\tilde{\omega}^{\dagger} \tilde{\omega}=1$. The unitarity of $U$ also implies $\tilde{\omega} \tilde{\omega}^{\dagger}+\omega \omega^{\dagger}=\mathbf{1}$.

One can show that (lepton and baryon-conserving) 4-fermion operators can only appear as hypercharge $Y=0, \pm 1$. This follows from an explicit enumeration of all possibilities. For the first category the 4 -fermion operators have to be dressed with $\omega, \tilde{\omega}$, to ensure hypercharge conservation. The following projections turn out to be useful

$$
\begin{array}{rlrl}
U P_{+} & =(\tilde{\omega}, 0), & U P_{12} & =(0, \tilde{\omega}) \\
U P_{21} & =(\omega, 0), & U P_{-}=(0, \omega)
\end{array}
$$

For instance, for the combination $\left(\bar{l} e_{R}\right)\left(\bar{q} u_{R}\right)$, one finds the two possibilites:

$$
\begin{aligned}
& \left(\bar{l} \tilde{\omega} e_{R}\right)\left(\bar{q} \omega u_{R}\right)=\left(\bar{l} U P_{12} \eta\right)\left(\bar{q} U P_{21} r\right) \\
& \left(\bar{l} \omega e_{R}\right)\left(\bar{q} \tilde{\omega} u_{R}\right)=\left(\bar{l} U P_{-} \eta\right)\left(\bar{q} U P_{+} r\right)
\end{aligned}
$$

For the $Y= \pm 14$-fermion operators, one achieves global neutral hypercharge dressing with the combinations $\omega, \omega$ and $\tilde{\omega}, \tilde{\omega}$. However, notice that the resulting operators are not independent, but rather related by hermitian conjugation.

\section{B.3 Fermion bilinear operators}

For this sector it is more convenient to work with the generic four combinations (signs are uncorrelated):

$$
P_{ \pm} \mathcal{A} P_{ \pm}
$$

where $P_{ \pm}=\frac{1}{2} \pm T_{3}$ are flavor projectors and $\mathcal{A}$ is any traceless, hermitean $S U(2)$ matrix (for instance $i L_{\mu}, \tau_{L}$, or $W_{\mu \nu}$ ). As such, $\mathcal{A}$ has only two independent elements and can be parametrized as

$$
\mathcal{A}=\left(\begin{array}{cc}
a & b \\
b^{\dagger} & -a
\end{array}\right) .
$$

Notice that Eq. (94) actually selects the different components of $\mathcal{A}$, namely

$$
\begin{aligned}
& P_{+} \mathcal{A} P_{+}=a P_{+}, \\
& P_{+} \mathcal{A} P_{-}=b P_{12}, \\
& P_{-} \mathcal{A} P_{+}=b^{\dagger} P_{21}, \\
& P_{-} \mathcal{A} P_{-}=-a P_{-},
\end{aligned}
$$

where

$$
\begin{aligned}
a & =\operatorname{tr}\left(T_{3} \mathcal{A}\right), \\
b & =\operatorname{tr}\left(P_{21} \mathcal{A}\right), \\
b^{\dagger} & =\operatorname{tr}\left(P_{12} \mathcal{A}\right),
\end{aligned}
$$


and $P_{i j}$ are the $S U(2)$ ladder operators. Inserting as many times Eq. (94) between fermion bilinears as needed, one can easily exhaust the list of operators for any dimension. Notice that the products of projectors have to be conveniently threaded using $P_{+} P_{+}=$ $P_{+}, P_{-} P_{-}=P_{-}$and $P_{+} P_{-}=P_{-} P_{+}=0$. For instance, consider NLO operators to the Yukawa terms $\bar{Q}_{L} U P_{ \pm} Q_{R}$. One has to consider operators stemming from

$$
\bar{Q}_{L} U\left[P_{ \pm} R_{\mu} P_{ \pm} R^{\mu} P_{ \pm}\right] Q_{R}
$$

where $R_{\mu}=U^{\dagger} D_{\mu} U$. Out of the 8 possibilities to connect the two projectors, only 6 are independent (up to h.c.), namely

$$
\begin{array}{cl}
\bar{Q}_{L} U P_{ \pm} Q_{R}\left\langle\tau_{L} L_{\mu}\right\rangle^{2}, & \bar{Q}_{L} U P_{ \pm} Q_{R}\left\langle L^{\mu} L_{\mu}\right\rangle \\
\bar{Q}_{L} U P_{12} Q_{R}\left\langle P_{21} R_{\mu}\right\rangle\left\langle\tau_{L} L^{\mu}\right\rangle, & \bar{Q}_{L} U P_{21} Q_{R}\left\langle P_{12} R_{\mu}\right\rangle\left\langle\tau_{L} L^{\mu}\right\rangle .
\end{array}
$$

Analogously, NLO operators to the left-handed vector current will come from

$$
\bar{Q}_{L} \gamma^{\mu} U\left[P_{ \pm} R_{\mu} P_{ \pm}\right] U^{\dagger} Q_{L}
$$

Out of the 4 possibilities, 3 are linearly independent (up to h.c.):

$$
i \bar{Q}_{L} \gamma_{\mu} Q_{L}\left\langle\tau_{L} L^{\mu}\right\rangle, \quad i \bar{Q}_{L} \gamma_{\mu} \tau_{L} Q_{L}\left\langle\tau_{L} L^{\mu}\right\rangle, \quad i \bar{Q}_{L} \gamma_{\mu} U P_{12} U^{\dagger} Q_{L}\left\langle P_{21} R^{\mu}\right\rangle
$$

\section{NLO operators in unitary gauge}

In order to get a clearer picture of the phenomenological consequences of the NLO operators, it is convenient to write them down in unitary gauge. In this gauge only physical particles are present, that is, $U=1$ and the Goldstone bosons are traded in for the longitudinal modes of the $W$ and $Z$ gauge bosons. In the following, we will rotate the $W_{\mu}^{a}$ and $B_{\mu}$ fields and express operators in terms of physical gauge bosons. An exception will be made with the operators $\mathcal{O}_{X U j}$, which are more conveniently expressed in terms of $W_{\mu \nu}^{3}$ and $B_{\mu}$, since they contribute to the oblique parameters $S, T$ and $U$.

The leading order Lagrangian $\mathcal{L}_{U}$ then reduces to the mass term for the gauge bosons and fermions:

$$
\mathcal{L}_{U}=\frac{v^{2}}{8}\left[2 g^{2} W_{\mu}^{+} W^{\mu-}+\left(g^{2}+g^{\prime 2}\right) Z_{\mu} Z^{\mu}\right]-v\left(\bar{u}_{L} Y_{u} u_{R}+\bar{d}_{L} Y_{d} d_{R}+\bar{e}_{L} Y_{e} e_{R}+\text { h.c. }\right)
$$

The leading custodial symmetry breaking operator (9) can be expressed as

$$
\mathcal{L}_{\beta_{1}}=-\frac{\beta_{1} v^{2}}{4}\left(g W_{\mu}^{3}-g^{\prime} B_{\mu}\right)^{2} \equiv-\frac{\beta_{1} v^{2}}{4}\left(g^{2}+g^{\prime 2}\right) Z_{\mu} Z^{\mu}
$$


Likewise, one can list the NLO operators for each class of section 4 (in the following we will omit those operators that in a general gauge do not contain the chiral field $U$ ):

$$
\begin{aligned}
& \mathcal{O}_{D 1}=\frac{1}{4}\left[2 g^{2} W_{\mu}^{+} W^{\mu-}+\left(g^{2}+g^{\prime 2}\right) Z_{\mu} Z^{\mu}\right]^{2} \\
& \mathcal{O}_{D 2}=\frac{1}{4}\left[g^{2}\left(W_{\mu}^{+} W_{\nu}^{-}+W_{\nu}^{+} W_{\mu}^{-}\right)+\left(g^{2}+g^{\prime 2}\right) Z_{\mu} Z_{\nu}\right]^{2} \\
& \mathcal{O}_{D 3}=\frac{1}{16}\left[\left(g^{2}+g^{\prime 2}\right) Z_{\mu} Z^{\mu}\right]^{2} \\
& \mathcal{O}_{D 4}=-\frac{1}{8}\left[\left(g^{2}+g^{\prime 2}\right) Z_{\mu} Z^{\mu}\right]\left[2 g^{2} W_{\nu}^{+} W^{-\nu}+\left(g^{2}+g^{\prime 2}\right) Z_{\nu} Z^{\nu}\right] \\
& \mathcal{O}_{D 5}=-\frac{1}{8}\left[\left(g^{2}+g^{\prime 2}\right) Z^{\mu} Z^{\nu}\right]\left[2 g^{2} W_{\mu}^{+} W_{\nu}^{-}+\left(g^{2}+g^{\prime 2}\right) Z_{\mu} Z_{\nu}\right]
\end{aligned}
$$

$$
\begin{array}{ll}
\mathcal{O}_{X U 1}=\frac{g^{\prime} g}{2} B^{\mu \nu} W_{\mu \nu}^{3} & \mathcal{O}_{X U 2}=\frac{g^{2}}{4} W_{\mu \nu}^{3} W^{3 \mu \nu} \\
\mathcal{O}_{X U 3}=\frac{g}{4} \varepsilon^{\mu \nu \lambda \rho}\left[g W_{\mu \nu}^{a} W_{\lambda}^{a}-g^{\prime} W_{\mu \nu}^{3} B_{\lambda}\right]\left[g^{\prime} B_{\rho}-g W_{\rho}^{3}\right] & \\
\mathcal{O}_{X U 4}=\frac{g^{\prime} g}{2} \varepsilon^{\mu \nu \lambda \rho} B_{\mu \nu} W_{\lambda \rho}^{3} & \mathcal{O}_{X U 5}=\frac{g^{2}}{4} \varepsilon^{\mu \nu \lambda \rho} W_{\mu \nu}^{3} W_{\lambda \rho}^{3} \\
\mathcal{O}_{X U 6}=\frac{g}{4}\left[g W_{\mu \nu}^{a} W^{a \mu}-g^{\prime} W_{\mu \nu}^{3} B^{\mu}\right]\left[g^{\prime} B^{\nu}-g W^{3 \nu}\right] &
\end{array}
$$
$\mathcal{O}_{\psi V 1}=-\frac{\sqrt{g^{2}+g^{\prime 2}}}{2} \bar{q} \gamma^{\mu} q Z_{\mu}$
$\mathcal{O}_{\psi V 2}=-\frac{\sqrt{g^{2}+g^{\prime 2}}}{2} \bar{q} \gamma^{\mu} T_{3} q Z_{\mu}$
$\mathcal{O}_{\psi V 3}=-\frac{g}{\sqrt{2}} \bar{u}_{L} \gamma^{\mu} d_{L} W_{\mu}^{+}$
$\mathcal{O}_{\psi V 4}=-\frac{\sqrt{g^{2}+g^{\prime 2}}}{2} \bar{u}_{R} \gamma^{\mu} u_{R} Z_{\mu}$
$\mathcal{O}_{\psi V 5}=-\frac{\sqrt{g^{2}+g^{\prime 2}}}{2} \bar{d}_{R} \gamma^{\mu} d_{R} Z_{\mu}$
$\mathcal{O}_{\psi V 6}=-\frac{g}{\sqrt{2}} \bar{u}_{R} \gamma^{\mu} d_{R} W_{\mu}^{+}$
$\mathcal{O}_{\psi V 7}=-\frac{\sqrt{g^{2}+g^{\prime 2}}}{2} \bar{l} \gamma^{\mu} l Z_{\mu}$
$\mathcal{O}_{\psi V 8}=-\frac{\sqrt{g^{2}+g^{\prime 2}}}{2} \bar{l} \gamma^{\mu} T_{3} l Z_{\mu}$
$\mathcal{O}_{\psi V 9}=-\frac{g}{\sqrt{2}} \bar{\nu}_{L} \gamma^{\mu} e_{L} W_{\mu}^{+}$
$\mathcal{O}_{\psi V 10}=-\frac{\sqrt{g^{2}+g^{\prime 2}}}{2} \bar{e}_{R} \gamma^{\mu} e_{R} Z_{\mu}$ 


$$
\begin{aligned}
& \mathcal{O}_{\psi S 1,2}=\frac{1}{2}\left(\bar{u}_{L} u_{R}, \bar{d}_{L} d_{R}\right)\left[2 g^{2} W_{\mu}^{+} W^{-\mu}+\left(g^{2}+g^{\prime 2}\right) Z_{\mu} Z^{\mu}\right] \\
& \mathcal{O}_{\psi S 3,4}=-\frac{g^{2}+g^{\prime 2}}{4}\left(\bar{u}_{L} u_{R}, \bar{d}_{L} d_{R}\right) Z_{\mu} Z^{\mu} \\
& \mathcal{O}_{\psi S 5}=-\frac{g \sqrt{g^{2}+g^{\prime 2}}}{2 \sqrt{2}} \bar{u}_{L} d_{R} W_{\mu}^{+} Z^{\mu} \quad \mathcal{O}_{\psi S 6}=-\frac{g \sqrt{g^{2}+g^{\prime 2}}}{2 \sqrt{2}} \bar{d}_{L} u_{R} W_{\mu}^{-} Z^{\mu} \\
& \mathcal{O}_{\psi S 7}=\frac{1}{2} \bar{e}_{L} e_{R}\left[2 g^{2} W_{\mu}^{+} W^{-\mu}+\left(g^{2}+g^{\prime 2}\right) Z_{\mu} Z^{\mu}\right] \\
& \mathcal{O}_{\psi S 8}=-\frac{g^{2}+g^{\prime 2}}{4} \bar{e}_{L} e_{R} Z_{\mu} Z^{\mu} \quad \mathcal{O}_{\psi S 9}=-\frac{g \sqrt{g^{2}+g^{\prime 2}}}{2 \sqrt{2}} \bar{\nu}_{L} e_{R} W_{\mu}^{+} Z^{\mu} \\
& \mathcal{O}_{\psi T 1}=-\frac{g \sqrt{g^{2}+g^{\prime 2}}}{2 \sqrt{2}} \bar{u}_{L} \sigma^{\mu \nu} d_{R} W_{\mu}^{+} Z_{\nu} \quad \mathcal{O}_{\psi T 2}=-\frac{g \sqrt{g^{2}+g^{\prime 2}}}{2 \sqrt{2}} \bar{d}_{L} \sigma^{\mu \nu} u_{R} W_{\mu}^{-} Z_{\nu} \\
& \mathcal{O}_{\psi T 3,4}=\frac{g^{2}}{2}\left(\bar{u}_{L} \sigma^{\mu \nu} u_{R}, \bar{d}_{L} \sigma^{\mu \nu} d_{R}\right) W_{\mu}^{+} W_{\nu}^{-} \\
& \mathcal{O}_{\psi T 5}=-\frac{g \sqrt{g^{2}+g^{\prime 2}}}{2 \sqrt{2}} \bar{\nu}_{L} \sigma^{\mu \nu} e_{R} W_{\mu}^{+} Z_{\nu} \quad \mathcal{O}_{\psi T 6}=\frac{g^{2}}{2} \bar{e}_{L} \sigma^{\mu \nu} e_{R} W_{\mu}^{+} W_{\nu}^{-} \\
& \mathcal{O}_{\psi X 1}=g_{s} \bar{u}_{L} \sigma^{\mu \nu} G_{\mu \nu} u_{R} \quad \mathcal{O}_{\psi X 2}=g_{s} \bar{d}_{L} \sigma^{\mu \nu} G_{\mu \nu} d_{R} \\
& \mathcal{O}_{\psi X 3}=g \bar{u}_{L} \sigma^{\mu \nu} u_{R}\left(\partial_{\mu} W_{\nu}^{3}+i g W_{\mu}^{+} W_{\nu}^{-}\right)+\sqrt{2} g \bar{d}_{L} \sigma^{\mu \nu} u_{R}\left(\partial_{\mu} W_{\nu}^{-}+i g W_{\mu}^{-} W_{\nu}^{3}\right) \\
& \mathcal{O}_{\psi X 4}=-g \bar{d}_{L} \sigma^{\mu \nu} d_{R}\left(\partial_{\mu} W_{\nu}^{3}+i g W_{\mu}^{+} W_{\nu}^{-}\right)+\sqrt{2} g \bar{u}_{L} \sigma^{\mu \nu} d_{R}\left(\partial_{\mu} W_{\nu}^{+}-i g W_{\mu}^{+} W_{\nu}^{3}\right) \\
& \mathcal{O}_{\psi X 5}=-g \bar{e}_{L} \sigma^{\mu \nu} e_{R}\left(\partial_{\mu} W_{\nu}^{3}+i g W_{\mu}^{+} W_{\nu}^{-}\right)+\sqrt{2} g \bar{\nu}_{L} \sigma^{\mu \nu} e_{R}\left(\partial_{\mu} W_{\nu}^{+}-i g W_{\mu}^{+} W_{\nu}^{3}\right) \\
& \mathcal{O}_{\psi X 6}=g^{\prime} \bar{u}_{L} \sigma^{\mu \nu} B_{\mu \nu} u_{R} \quad \mathcal{O}_{\psi X 7}=g^{\prime} \bar{d}_{L} \sigma^{\mu \nu} B_{\mu \nu} d_{R} \quad \mathcal{O}_{\psi X 8}=g^{\prime} \bar{e}_{L} \sigma^{\mu \nu} B_{\mu \nu} e_{R}
\end{aligned}
$$
$\mathcal{O}_{L L 6}=\bar{q} \gamma^{\mu} T_{3} q \bar{q} \gamma_{\mu} T_{3} q$
$\mathcal{O}_{L L 7}=\bar{q} \gamma^{\mu} T_{3} q \bar{q} \gamma_{\mu} q$
$\mathcal{O}_{L L 8}=\bar{q}_{\alpha} \gamma^{\mu} T_{3} q_{\beta} \bar{q}_{\beta} \gamma_{\mu} T_{3} q_{\alpha}$
$\mathcal{O}_{L L 9}=\bar{q}_{\alpha} \gamma^{\mu} T_{3} q_{\beta} \bar{q}_{\beta} \gamma_{\mu} q_{\alpha}$
$\mathcal{O}_{L L 10}=\bar{q} \gamma^{\mu} T_{3} q \bar{l} \gamma_{\mu} T_{3} l$
$\mathcal{O}_{L L 11}=\bar{q} \gamma^{\mu} T_{3} q \bar{l} \gamma_{\mu} l$
$\mathcal{O}_{L L 12}=\bar{q} \gamma^{\mu} q \bar{l} \gamma_{\mu} T_{3} l$
$\mathcal{O}_{L L 13}=\bar{q} \gamma^{\mu} T_{3} l \bar{l} \gamma_{\mu} T_{3} q$
$\mathcal{O}_{L L 14}=\bar{q} \gamma^{\mu} T_{3} l \bar{l} \gamma_{\mu} q$
$\mathcal{O}_{L L 15}=\bar{l} \gamma^{\mu} T_{3} l \bar{l} \gamma_{\mu} T_{3} l$
$\mathcal{O}_{L L 16}=\bar{l} \gamma^{\mu} T_{3} l \bar{l} \gamma_{\mu} l$ 


$$
\begin{array}{rlrl}
\mathcal{O}_{L R 10} & =\bar{q} \gamma^{\mu} T_{3} q \bar{u}_{R} \gamma_{\mu} u_{R} & & \mathcal{O}_{L R 11}=\bar{q} \gamma^{\mu} T^{A} T_{3} q \bar{u}_{R} \gamma_{\mu} T^{A} u_{R} \\
\mathcal{O}_{L R 12}=\bar{q} \gamma^{\mu} T_{3} q \bar{d}_{R} \gamma_{\mu} d_{R} & & \mathcal{O}_{L R 13}=\bar{q} \gamma^{\mu} T^{A} T_{3} q \bar{d}_{R} \gamma_{\mu} T^{A} d_{R} \\
\mathcal{O}_{L R 14}=\bar{u}_{R} \gamma^{\mu} u_{R} \bar{l}_{\mu} T_{3} l & & \mathcal{O}_{L R 15}=\bar{d}_{R} \gamma^{\mu} d_{R} \bar{l} \gamma_{\mu} T_{3} l \\
\mathcal{O}_{L R 16}=\bar{q} \gamma^{\mu} T_{3} q \bar{e}_{R} \gamma_{\mu} e_{R} & & \mathcal{O}_{L R 17}=\bar{l} \gamma^{\mu} T_{3} l \bar{e}_{R} \gamma_{\mu} e_{R} \\
\mathcal{O}_{L R 18}=\bar{q} \gamma^{\mu} T_{3} l \bar{e}_{R} \gamma_{\mu} d_{R} & &
\end{array}
$$

$$
\begin{aligned}
\mathcal{O}_{S T 5} & =\bar{u}_{L} u_{R} \bar{d}_{L} d_{R} & \mathcal{O}_{S T 6} & =\bar{d}_{L} u_{R} \bar{u}_{L} d_{R} \\
\mathcal{O}_{S T 7} & =\bar{u}_{L} T^{A} u_{R} \bar{d}_{L} T^{A} d_{R} & \mathcal{O}_{S T 8} & =\bar{d}_{L} T^{A} u_{R} \bar{u}_{L} T^{A} d_{R} \\
\mathcal{O}_{S T 9} & =\bar{u}_{L} u_{R} \bar{e}_{L} e_{R} & \mathcal{O}_{S T 10} & =\bar{d}_{L} u_{R} \bar{\nu}_{L} e_{R} \\
\mathcal{O}_{S T 11} & =\bar{u}_{L} \sigma^{\mu \nu} u_{R} \bar{e}_{L} \sigma_{\mu \nu} e_{R} & \mathcal{O}_{S T 12} & =\bar{d}_{L} \sigma^{\mu \nu} u_{R} \bar{\nu}_{L} \sigma_{\mu \nu} e_{R}
\end{aligned}
$$

$$
\begin{aligned}
\mathcal{O}_{F Y 1} & =\bar{u}_{L} u_{R} \bar{u}_{L} u_{R} & \mathcal{O}_{F Y 2} & =\bar{u}_{L} T^{A} u_{R} \bar{u}_{L} T^{A} u_{R} \\
\mathcal{O}_{F Y 3} & =\bar{d}_{L} d_{R} \bar{d}_{L} d_{R} & \mathcal{O}_{F Y 4} & =\bar{d}_{L} T^{A} d_{R} \bar{d}_{L} T^{A} d_{R} \\
\mathcal{O}_{F Y 5} & =\bar{d}_{L} d_{R} \bar{u}_{R} u_{L} & \mathcal{O}_{F Y 6} & =\bar{d}_{L} T^{A} d_{R} \bar{u}_{R} T^{A} u_{L} \\
\mathcal{O}_{F Y 7} & =\bar{d}_{L} d_{R} \bar{e}_{L} e_{R} & \mathcal{O}_{F Y 8} & =\bar{d}_{L} \sigma^{\mu \nu} d_{R} \bar{e}_{L} \sigma_{\mu \nu} e_{R} \\
\mathcal{O}_{F Y 9} & =\bar{e}_{L} e_{R} \bar{u}_{R} u_{L} & \mathcal{O}_{F Y 10} & =\bar{e}_{L} e_{R} \bar{e}_{L} e_{R} \\
\mathcal{O}_{F Y 11} & =\bar{e}_{L} d_{R} \bar{u}_{R} \nu_{L} & &
\end{aligned}
$$

\section{Acknowledgements}

We thank Gino Isidori for comments on the manuscript and useful discussions. We thank Witold Skiba for drawing our attention to ref. [18]. This work was performed in the context of the ERC Advanced Grant project 'FLAVOUR' (267104) and was supported in part by the DFG cluster of excellence 'Origin and Structure of the Universe'.

\section{References}

[1] D. B. Kaplan, H. Georgi and S. Dimopoulos, Phys. Lett. B 136, 187 (1984). 
[2] G. F. Giudice, C. Grojean, A. Pomarol and R. Rattazzi, JHEP 0706, 045 (2007) [arXiv:hep-ph/0703164]; R. Contino, C. Grojean, M. Moretti, F. Piccinini and R. Rattazzi, JHEP 1005, 089 (2010) [arXiv:1002.1011 [hep-ph]]; R. Contino, arXiv:1005.4269 [hep-ph]; R. Contino, Nuovo Cim. C 32N3-4, 11 (2009) [arXiv:0908.3578 [hep-ph]].

[3] H. Georgi, D. B. Kaplan and P. Galison, Phys. Lett. B 143, 152 (1984).

[4] ATLAS Collaboration, arXiv:1202.1408 [hep-ex].

[5] CMS Collaboration, arXiv:1202.1488 [hep-ex].

[6] T. Appelquist and C. W. Bernard, Phys. Rev. D 22, 200 (1980).

[7] S. Weinberg, Physica A 96, 327 (1979).

[8] A. C. Longhitano, Phys. Rev. D 22, 1166 (1980).

[9] A. C. Longhitano, Nucl. Phys. B 188, 118 (1981).

[10] T. Appelquist and G. H. Wu, Phys. Rev. D 48, 3235 (1993) [arXiv:hep-ph/9304240].

[11] T. Appelquist, M. J. Bowick, E. Cohler and A. I. Hauser, Phys. Rev. D 31, 1676 (1985).

[12] A. I. Hauser, Ph. D. Thesis, University of Yale, 1985.

[13] R. D. Peccei and X. Zhang, Nucl. Phys. B 337, 269 (1990).

[14] E. Bagan, D. Espriu and J. Manzano, Phys. Rev. D 60, 114035 (1999) [arXiv:hep$\mathrm{ph} / 9809237]$.

[15] J. A. Manzano Flecha, arXiv:hep-ph/0208068.

[16] A. Dobado, D. Espriu and M. J. Herrero, Phys. Lett. B 255, 405 (1991); A. F. Falk, M. E. Luke and E. H. Simmons, Nucl. Phys. B 365, 523 (1991); A. De Rujula, M. B. Gavela, P. Hernandez and E. Masso, Nucl. Phys. B 384, 3 (1992); D. Espriu and M. J. Herrero, Nucl. Phys. B 373, 117 (1992); S. Dawson and G. Valencia, Phys. Rev. D 49, 2188 (1994) [arXiv:hep-ph/9308248]; P. Hernandez and F. J. Vegas, Phys. Lett. B 307, 116 (1993) [arXiv:hep-ph/9212229]; C. P. Burgess and D. London, Phys. Rev. Lett. 69, 3428 (1992).

[17] A. Nyffeler and A. Schenk, Phys. Rev. D 62, 113006 (2000) [arXiv:hep-ph/9907294].

[18] C. Grojean, W. Skiba and J. Terning, Phys. Rev. D 73, 075008 (2006) [arXiv:hep$\mathrm{ph} / 0602154]$.

[19] S. Weinberg, Phys. Rev. 166, 1568 (1968); J. Gasser and H. Leutwyler, Annals Phys. 158, 142 (1984). 
[20] M. E. Peskin and T. Takeuchi, Phys. Rev. D 46, 381 (1992).

[21] M. J. Herrero and E. Ruiz Morales, Nucl. Phys. B 418, 431 (1994) [arXiv:hep$\mathrm{ph} / 9308276]$.

[22] A. Manohar and H. Georgi, Nucl. Phys. B 234, 189 (1984); R. S. Chivukula, arXiv:hep-ph/0011264.

[23] C. Arzt, M. B. Einhorn and J. Wudka, Nucl. Phys. B 433, 41 (1995) [hep$\mathrm{ph} / 9405214]$.

[24] B. Grzadkowski, M. Iskrzynski, M. Misiak and J. Rosiek, JHEP 1010, 085 (2010) [arXiv:1008.4884 [hep-ph]].

[25] W. Buchmüller and D. Wyler, Nucl. Phys. B 268, 621 (1986).

[26] S. Weinberg, Phys. Rev. Lett. 43, 1566 (1979); F. Wilczek and A. Zee, Phys. Rev. Lett. 43, 1571 (1979); L. F. Abbott and M. B. Wise, Phys. Rev. D 22, 2208 (1980).

[27] J. Hirn and J. Stern, Phys. Rev. D 73, 056001 (2006) [arXiv:hep-ph/0504277].

[28] J. F. Donoghue, E. Golowich and B. R. Holstein, Camb. Monogr. Part. Phys. Nucl. Phys. Cosmol. 2, 1 (1992).

[29] G. Isidori, PoS C D09, 073 (2009) [arXiv:0911.3219 [hep-ph]]. 